\title{
Designing a strategyproof spot market mechanism with many traders: twenty-two steps to Walrasian equilibrium
}

\author{
Peter J. Hammond ${ }^{1}$
}

Received: 31 December 2015 / Accepted: 1 April 2016 / Published online: 4 May 2016 (C) The Author(s) 2016. This article is published with open access at Springerlink.com

\begin{abstract}
To prove their Walrasian equilibrium existence theorem, Arrow and Debreu (Econometrica 22(3):265-290, 1954) devised an abstract economy that Shapley and Shubik (J Polit Econ 85:937-968, 1977) criticized as a market game because, especially with untrustworthy traders, it fails to determine a credible outcome away from equilibrium. All this earlier work also postulated a Walrasian auctioneer with complete information about traders' preferences and endowments. To ensure credible outcomes, even in disequilibrium, warehousing is introduced into a multistage market game. To achieve Walrasian outcomes in a large economy with incomplete information, even about traders' endowments, a strategyproof demand revelation mechanism is considered and then extended to include warehousing.
\end{abstract}

Keywords Market design · Demand revelation - Strategyproofness · Hidden endowments · Warehousing

JEL Classification C72 · D41 · D47 · D51

\section{Introduction and background}

\subsection{Perfectly competitive spot markets}

Following a literature that extends from Arrow (1951) to Hammond (2011) and beyond, the equilibrium allocations that result from a perfectly competitive spot market system have efficiency and welfare properties that are fairly well understood. So are sufficient

Peter J. Hammond

p.j.hammond@warwick.ac.uk

1 Department of Economics, University of Warwick, Coventry CV4 7AL, UK 
conditions for Walrasian equilibrium in such a system to exist. In fact, given any fixed status quo allocation, such a system, when combined with a suitable "pre-distributive" policy for distributing any potential gains, can produce an equilibrium allocation that is Pareto superior to the status quo. That is, except in the uninteresting special case when the status quo is already Pareto efficient.

Yet ever since Walras's own writings concerning his concepts of the auctioneer and tâtonnement, economists have been vexed by the problem of designing some kind of mechanism or process to ensure that Walrasian equilibrium in a spot market system could actually be attained.

\subsection{Walrasian tâtonnement}

Originally the question that was asked is whether a tâtonnement process for adjusting prices in response to excess demand could converge to a Walrasian equilibrium. Except in special cases such as when all commodities are "gross substitutes", the conclusions were generally rather unsatisfactory - see, for example, the survey by Hahn (1993). Even the tâtonnement process itself required each economic agent to report the appropriate value of its excess demand function truthfully; there was no discussion of any strategic considerations.

\subsection{The Arrow-Debreu "abstract economy"}

In the standard definition of a non-cooperative game, each player's feasible set of strategies is independent of the other players' strategy choices. By contrast, Debreu (1952) introduced the notion of a "generalized game" where, by definition, each player's feasible set of strategies may depend on the other players' strategy choices. In their classic paper on existence of Walrasian equilibrium, Arrow and Debreu (1954) constructed an "abstract economy" as a generalized game which confronts the agents in an economy, who choose their own net trade vectors, with an auctioneer who chooses a price vector. Moreover, the economic agents are limited to choosing respective net trade vectors that satisfy their budget constraint, which is determined by the auctioneer's choice of price vector.

This generalized game was never intended to be a fully specified model of how any market system, realistic or artificial, could actually function. Instead, it merely served as a device allowing Debreu's (1952) existence theorem to be applied. This existence theorem relies on arguments that probably owe more to Glicksberg's (1952) method of proving existence of Nash equilibrium in pure strategies, when the set of pure strategies is convex, than to Nash's (1950) own proof that a mixed strategy equilibrium exists when there is a finite set of pure strategies.

\subsection{Myerson's coordination device}

One obvious issue in using the Arrow-Debreu abstract economy is its essential reliance on Nash equilibrium as the solution concept. In particular, each trader must anticipate 
the auctioneer's choice of price vector simply in order to be sure of satisfying the budget constraint. Following the discussion by Polak (1999) of equilibrium in general games, restrictive epistemic conditions are required in order to ensure that the players can reach a pure strategy Nash equilibrium corresponding to a Walrasian equilibrium.

Indeed, a robust market system should be able to handle traders who know little, and care even less, about one another's preferences, endowments, and trading strategies. Formally, following Myerson (1982), one can consider a mechanism where a completely informed principal suggests a Nash equilibrium of the generalized game, which is then a Walrasian equilibrium of the economy. Nevertheless, the latter part of this paper will move toward what seems the more relevant case where, in the spirit of Harsanyi (1967-1968), the principal guides the agents to a particular Bayesian Nash equilibrium in a game with incomplete information.

\subsection{The Shapley-Shubik critique}

Let me quote from footnote 1 of Shapley and Shubik (1977), where they discuss the market game that Arrow and Debreu (1954) use to prove existence of Walrasian equilibrium: ${ }^{1}$

But as a descriptive model [t]his game shares the defect of the Walras exchange model of being ill defined, or unrealistically defined, away from equilibrium. Indeed, if only one agent departs from equilibrium, he is presumed to be able to buy and sell at the stated prices, announced by an added fictive player whose objective is to minimize excess demand. But there is no explanation of how the excess demand thereby created is to be satisfied-unless it is out of the bottomless warehouses of the fictive player.

\subsection{The warehousing remedy}

In mentioning "warehouses", as well as in introducing their well known model with trading posts, Shapley and Shubik (1977) implicitly suggest a possible remedy for this serious deficiency in the Arrow-Debreu market game. ${ }^{2}$ This is to allow the Walrasian auctioneer to combine three functions:

1. the traditional Walrasian auctioneer, who chooses a price vector;

2. a coordinator who, like Myerson's (1982) principal, recommends-even mandates-both demand and supply vectors for each economic agent subject to

\footnotetext{
${ }^{1}$ The preceding sentence of their footnote reads as follows: "In his important early paper, Debreu (1952) represents the Walras exchange model as a game in strategic form for the technical purpose of applying a general existence theorem." Yet Debreu (1952) refers to the Walras exchange model only when citing the (then) forthcoming paper by Arrow and Debreu. So I have changed "his" to "[t]his" in quoting the remainder of the footnote.

2 Thanks to Kenneth Judd for reminding me that Keisler $(1995,1996)$ uses inventories to accommodate mismatches between demand and supply during a non-tâtonnement process of quantity adjustment to a Walrasian equilibrium.
} 
the incentive constraints that these vectors must be what the agents themselves would be willing to choose given the chosen price vector;

3. a warehouse manager, who manages a system that takes in all the agents' offered supplies up to their mandated levels, and then allocates whatever total supplies have been made available in order to meet agents' demands as far as possible.

The key idea is to allow supplies to be collected before attempting to meet any demands. In order to do this, one has to consider a game in two stages, as will be done in Sect. 4.

\subsection{From Vickrey and Harsanyi toward market design}

Vickrey (1961) proposes a second-price auction for a simple market in which one committed seller plans to sell one indivisible item to one of several competing buyers. The model anticipates Harsanyi's (1967-1968) work on adapting Nash's theory of noncooperative strategic games to accommodate incomplete information. Wilson (1985) and others have been able to use Vickrey's ideas in developing a theory of "double auctions" for a single market that confronts several competing potential sellers, each with one unit of a homogenous commodity they might sell, with several competing potential buyers, each able to buy at most one of those units.

Ideas building on Vickrey's and Harsanyi's theories have also been widely applied to the design of auction markets for a wide range of products. These include licences in many countries to use parts of the electromagnetic spectrum in order to transmit information, as well as "Certificates of Entitlement" for owning a car in Singapore.

\subsection{Hidden endowments}

To the author's knowledge, however, nobody has yet faced up to the task of designing a game of incomplete information whose outcomes can reasonably be expected to generate a Pareto efficient allocation in a general multimarket system with many traders. This is especially true when, as in the work by Postlewaite (1979) and by Hurwicz et al. (1995), there is incomplete information regarding traders' endowments, and traders can consume whatever they conceal.

Indeed, it was assumed in Hammond (1979), as well as in later work surveyed in Hammond (2011, Sections 14-15), that agents would restrict themselves to reporting types for which the allocation mechanism specified a net trade that was feasible given the true endowment vector. The only sanction facing somebody who violated this constraint was that, if misreporting their type led to a supply contract being broken, they would be required to keep reporting a different type until the feasibility constraint was satisfied and a supply contract was reached that could be honoured. Even this sanction was left entirely implicit. For an alternative in a two-period setting, where Walrasian equilibrium may be unattainable, see Hammond (1992).

In the case of financial markets, the need for some radical system re-design seems entirely evident given events that led to the recent "Great Recession" in most Western economies. At this stage, however, it would seem highly premature to suggest how to reform financial markets. For now, the much less ambitious task of designing a gen- 
eral spot market system that can implement Walrasian allocations seems challenging enough.

After reviewing a few key approaches to the issues mentioned above, the main purpose of the paper is to propose a demand revelation mechanism with warehousing that attempts to meet the suggested requirements.

\subsection{Outline of paper}

Section 2 introduces the basic definitions, notation, and assumptions for a class of pure exchange economies. Thereafter, Sect. 3 presents a significant variation of the generalized game that Arrow and Debreu (1954) used to define their abstract economy. In particular, before the traders are required to announce their demands, they are informed of the price vector chosen by the auctioneer or market organizer. Then each trader's strategy becomes, rather than the simple demand vector it was in Arrow and Debreu's generalized game, an entire demand function of the price that the rules of the game require always to satisfy the budget constraint. This extension to functions makes the traders' strategy sets independent of the auctioneer's choice of any one particular price vector. Hence, this variation produces a game rather than a generalized game.

So far there have been no explicit incentives for traders to fulfil their supply contracts. Following the key idea of Shapley and Shubik (1977), Sect. 4 considers the implications of requiring all supplies to be collected in a system of warehouses or trading posts, before being released to meet traders' demands as far as possible. Traders can be deterred from default by excluding all those who break their contracts from any access to warehoused supplies.

Next, Sect. 5 turns attention to the case when the market organizer is incompletely informed of both traders' preferences and their endowments. In this setting, it is well known that there is a strategyproof allocation mechanism guaranteeing Pareto efficient outcomes only if either allocations close to the dictatorial or oligarchic extremes are selected, or else there is an infinite population of traders. Contrast, for example, the results of Myerson and Satterthwaite (1983), Serizawa (2002) and Serizawa and Weymark (2003) with those of Hammond (1979) and Guesnerie (1998). Accordingly, Sect. 5 introduces the notion of a statistical continuum economy. Earlier work, including Hart et al. (1974), considers just the distribution of traders' characteristics as well as their net demand vectors. Here, by contrast, a joint distribution over traders' labels as well as their characteristics, net demand vectors, etc. is considered. This relaxes some obvious symmetry conditions.

Then, building on previous ideas in Hammond (1979), Sect. 6 constructs a strategyproof demand revelation mechanism that implements Walrasian equilibrium. This works, however, only for the case when traders ultimately limit themselves to revealing demands which guarantee that they can honour their supply contracts. This leaves open the problem of providing more effective incentives to dissuade traders from revealing net demand correspondences which create the possibility that they may be asked to supply more of some goods than their true endowments. To overcome this problem, Sect. 7 follows Sect. 4 in combining warehouses with a two-stage market system. Each stage uses a demand revelation mechanism like that in Sect. 6. 
In Sect. 8, an extensive form game in twenty-two steps is formally defined. It involves infinitely many traders and one market organizer or principal who also acts as recording clerk, statistician, auctioneer, market equilibrator, and warehouse manager. The game implements Walrasian equilibrium when backward induction is used to eliminate dominated strategies recursively in relevant continuation subgames of incomplete information, as well as in the game as a whole. ${ }^{3}$ In this sense, even when traders' endowments as well as their preferences are unobservable, appropriate incentives are created for individuals to reveal their true Walrasian demand correspondences and also to deliver whatever supplies are required to reach Walrasian equilibrium.

The main results, along with their limitations, are reviewed in the concluding Sect. 9, which also contains some ideas for future research concerning extensions beyond spot markets in a pure exchange economy.

Appendix 1 sets out some useful selected results concerning probability measures on Polish spaces-i.e. complete and separable metric spaces. Appendix 2 shows how the space of suitably regular closed graph demand correspondences can be given a metric that makes it a Polish space. Finally, Appendix 3 offers a proof that a market clearing equilibrium price exists in our setting where a countably infinite set of traders with random labels is required to submit demand correspondences satisfying the regularity conditions imposed earlier.

\section{A pure exchange economy}

\subsection{Commodities and their prices}

Let $G$ denote a finite set of commodities, with typical member $g$, which traders may be able to exchange. The commodity space whose members are vectors such as $x=$ $\left(x_{g}\right)_{g \in G}$ will then be denoted by $\mathbb{R}^{G}$. Let $1^{G}=(1,1, \ldots, 1) \in \mathbb{R}_{++}^{G}$ denote the specific vector that has all its components equal to 1 .

Let $p=\left(p_{g}\right)_{g \in G} \in \mathbb{R}_{+}^{G} \backslash\{0\}$ denote a typical semi-positive price vector, and

$$
P:=\left\{p \in \mathbb{R}_{+}^{G} \mid \sum_{g \in G} p_{g}=1\right\}
$$

the unit simplex of normalized price vectors, with relative interior

$$
P^{0}:=\left\{p \in \mathbb{R}_{++}^{G} \mid \sum_{g \in G} p_{g}=1\right\}
$$

and relative boundary bd $P:=P \backslash P^{0}$.

\footnotetext{
3 Like orthodox subgames, subgames of incomplete information arise as continuations of an extensive form game after some initial moves have been made. Unlike orthodox subgames, but like games of incomplete information, the precise continuation subgame being played typically depends on players' hidden types.
} 


\subsection{Traders and their endowments}

For our initial discussion of a game of complete information with warehousing, we will consider a pure exchange economy with a finite set $I$ of traders. Each trader $i \in I$ is assumed to have a fixed endowment vector $e^{i} \in \mathbb{R}_{+}^{G}$. We also assume that the commodity set $G$ excludes irrelevant goods $g$ that cannot be traded because their total endowment $\sum_{i \in I} e_{g}^{i}=0$. Accordingly, we assume that $\sum_{i \in I} e^{i} \in \mathbb{R}_{++}^{G}$.

In the early part of the paper, the set $G$ and the associated commodity space $\mathbb{R}^{G}$ will be treated as fixed. Once the demand revelation mechanism of Sect. 6 is being considered, however, the trading space of exchangeable commodities is allowed to become the variable set of goods for which a non-null set of traders claim to have positive endowments.

\subsection{Net trades}

For any trader $i \in I$, a typical net trade vector will be denoted by $z^{i} \in \mathbb{R}^{G}$, with components $z_{g}^{i}$ for $g \in G$. This vector can be decomposed uniquely as $z^{i}=x^{i}-y^{i}$ where $x^{i} \in \mathbb{R}_{+}^{G}$ and $y^{i} \in \mathbb{R}_{+}^{G}$ are separate non-negative demand and supply vectors with $x_{g}^{i}=\max \left\{z_{g}^{i}, 0\right\}$ and $y_{g}^{i}=\max \left\{-z_{g}^{i}, 0\right\}$ for all $g \in G$. Using standard lattice notation, one can then write

$$
x^{i}=z^{i} \vee 0 \text { and } y^{i}=-\left(z^{i} \wedge 0\right)=\left(-z^{i}\right) \vee 0
$$

\subsection{Feasible sets}

Assume that each trader $i \in I$, given the endowment vector $e^{i} \in \mathbb{R}_{+}^{G}$, is able:

1. to supply any non-negative commodity vector $y^{i}$ that belongs to the supply set $Y^{i}:=\left\{y^{i} \in \mathbb{R}_{+}^{G} \mid y^{i} \leqq e^{i}\right\}$

2. to demand any non-negative commodity vector $x^{i}$ that belongs to the consumption set $\mathbb{R}_{+}^{G}$.

This leads to the closed feasible set $Z^{i}:=\left\{z \in \mathbb{R}_{+}^{G} \mid z \geqq-e^{i}\right\}=\mathbb{R}_{+}^{G}+\left\{-e^{i}\right\}$ of net trade vectors $z^{i}=x^{i}-y^{i}$ with $x^{i} \in \mathbb{R}_{+}^{G}$ and $y^{i} \leqq e^{i}$.

Taking into account the resource balance constraint $\sum_{i \in I} x^{i} \leqq \sum_{i \in I} y^{i}$ with free disposal, it follows that one has the following three compact subsets of the Cartesian product set $\left(\mathbb{R}_{+}^{G}\right)^{I}$ whose dimension is the product \#I * \#G:

1. $Y^{I}:=\left\{y^{I} \in\left(\mathbb{R}_{+}^{G}\right)^{I} \mid y^{i} \leqq e^{i}\right.$ (all $\left.\left.i \in I\right)\right\}$ of feasible supply allocations;

2. $X^{I}:=\left\{x^{I} \in\left(\mathbb{R}_{+}^{G}\right)^{I} \mid \sum_{i \in I} x^{i} \leqq \sum_{i \in I} e^{i}\right\}$ of feasible demand allocations;

3. $Z^{I}:=\left\{z^{I} \in X^{I}-Y^{I} \mid \sum_{i \in I} z^{i} \leqq 0\right\}$ of feasible net trade allocations with free disposal.

Compactness of these three sets helps guarantee that a Walrasian equilibrium exists. 


\subsection{Preferences}

Assume that each trader $i \in I$ has a continuous, convex, and strictly monotone preference ordering $\succsim^{i}$ over consumption vectors $c^{i} \in \mathbb{R}_{+}^{G}$. It is well known that any such $\succsim^{i}$ can be represented by a continuous quasi-concave utility function $u^{i}: \mathbb{R}_{+}^{G} \rightarrow \mathbb{R}$ which is strictly increasing. For example, one can construct

$$
u^{i}\left(c^{i}\right):=\inf \left\{\gamma \in \mathbb{R} \mid \gamma 1^{G} \succsim^{i} c^{i}\right\}
$$

\section{Formulating an Arrow-Debreu game}

\subsection{An ordinary market game}

The following reformulation of the Arrow-Debreu abstract economy defines an ordinary strategic game, rather than a "generalized game" in the sense of Debreu (1952). In addition to the set of traders $i \in I$, there is one extra "fictive" player 0 whose role is that of the "Walrasian auctioneer".

Unlike Debreu's (1952) generalized game, our game has a non-trivial extensive form. Specifically, player 0 moves first and chooses a price vector $p \in P^{0}$. Each trader $i \in I$ then observes this choice of $p$, which determines that trader's endowment constrained budget set

$$
B\left(p ; e^{i}\right):=\left\{z \in \mathbb{R}^{G} \mid z \geqq-e^{i} \text { and } p z \leq 0\right\}
$$

of net trade vectors that are permissible choices for $i$ at the next stage of the game. Thus, for each trader $i \in I$, a feasible strategy, which we denote by $\mathfrak{z}^{i}$, is a net demand function $P^{0} \ni p \mapsto z^{i}(p) \in B\left(p ; e^{i}\right)$ whose value at each price $p \in P^{0}$ is a net trade vector $z^{i} \in B\left(p ; e^{i}\right)$. Hence, $\mathfrak{z}^{i}$ is a selection from trader $i$ 's endowment constrained budget correspondence $P^{0} \ni p \mapsto B\left(p ; e^{i}\right) \subset Z^{i}$. Note that trader $i$ 's feasible strategy set is the Cartesian product

$$
\mathfrak{B}^{i}:=\prod_{p \in P^{0}} B\left(p ; e^{i}\right)
$$

In this game, the typical strategy profile is a combination

$$
\left(p, \mathfrak{z}^{I}\right) \in P^{0} \times \prod_{i \in N} \mathfrak{B}^{i}
$$

of a price vector $p \in P^{0}$ and a profile $\mathfrak{z}^{I}=\left(\mathfrak{z}^{i}\right)_{i \in I} \in \prod_{i \in I} \mathfrak{B}^{i}$ of traders' net demand functions. Player 0's pay-off is taken to be

$$
v^{0}\left(p, \mathfrak{z}^{I}\right):=-\left\|\sum_{i \in I} z^{i}(p)\right\|=-\left(\sum_{g \in G}\left[\sum_{i \in I} z_{g}^{i}(p)\right]^{2}\right)^{1 / 2}
$$


-i.e. minus the Euclidean norm of the aggregate net demand induced by this strategy profile. Finally, each trader $i$ 's pay-off is taken to be

$$
v^{i}\left(p, \mathfrak{z}^{I}\right):=u^{i}\left(z^{i}(p)+e^{i}\right)
$$

\subsection{The case of strictly convex preferences}

Consider the special case where all traders' preferences are strictly convex-or equivalently, when their ordinal utility functions are strictly quasi-concave. This assumption implies that each trader $i \in I$ will have a single-valued Walrasian net demand function $\overline{\mathfrak{z}}^{i} \in \mathfrak{B}^{i}$ in the form of a mapping

$$
P^{0} \ni p \mapsto \bar{z}^{i}(p) \in B\left(p ; e^{i}\right)
$$

satisfying

$$
\left\{\bar{z}^{i}(p)\right\}=\arg \max _{z}\left\{u^{i}\left(z+e^{i}\right) \mid z \in B\left(p ; e^{i}\right)\right\}
$$

Then, in the subgame that follows the auctioneer's choice of any price vector $p \in P^{0}$, where each trader $i \in I$ is required to choose any net demand vector $z^{i} \in B\left(p ; e^{i}\right)$, it follows that trader $i$ 's unique dominant strategy is $\bar{z}^{i}(p)$.

With such single-valued net demand functions, the price vector $\bar{p} \in P^{0}$ is a Walrasian equilibrium just in case $\sum_{i \in I} \bar{z}^{i}(\bar{p})=0$. Now, applying backward induction shows that the strategy combination $(\bar{p}, \overline{\mathfrak{z}})$ is a subgame perfect Nash equilibrium if and only if:

1. each trader $i$ 's equilibrium strategy $\overline{\mathfrak{z}}^{i}$ is the Walrasian net demand function $p \mapsto$ $\bar{z}^{i}(p)$ defined by (9);

2. $\bar{p}$ is a Walrasian equilibrium price vector that, because of (6), gives player 0 the global maximum pay-off $v^{0}\left(\bar{p}, \overline{\mathfrak{z}}^{I}\right)=0$.

\subsection{The case of demand correspondences}

When trader $i$ 's preferences are not strictly convex, however, there is typically a Walrasian net demand correspondence or multifunction $\overline{\mathfrak{Z}}^{i}$ whose set value is defined by

$$
P^{0} \ni p \mapsto Z\left(p ; \overline{\mathfrak{Z}}^{i}\right):=\arg \max _{z}\left\{u^{i}\left(z+e^{i}\right) \mid z \in B\left(p ; e^{i}\right)\right\}
$$

Each set value is generally not a singleton.

In this case, a Walrasian equilibrium is a combination $\left(\bar{p}, \bar{z}^{I}\right)$ of a price vector $\bar{p} \in P^{0}$ and an allocation or profile $\bar{z}^{I}=\left(z^{i}\right)_{i \in I} \in\left(\mathbb{R}^{G}\right)^{I}$ of net trade vectors, one for each trader $i \in I$, satisfying both $\sum_{i \in I} \bar{z}^{i}=0$ and $\bar{z}^{i} \in Z\left(\bar{p} ; \overline{\mathfrak{Z}}^{i}\right)$ for all $i \in I$.

Even when they are evaluated at an equilibrium price vector $\bar{p} \in P^{0}$, the typical profile $z^{I}$ of net trade vectors selected from the value $\bar{z}^{I}(\bar{p}) \in \prod_{i \in I} Z\left(\bar{p} ; \overline{\mathfrak{Z}}^{i}\right)$ of the Cartesian product of traders' Walrasian net demand correspondences will fail to satisfy the market clearing requirement that $\sum_{i \in I} z^{i}=0$. For this reason, traders' realized net demands need to be more coordinated in order to implement a Walrasian equilibrium as a subgame perfect Nash equilibrium. 
Indeed, consider any particular Walrasian equilibrium $\left(\bar{p}, \bar{z}^{I}\right)$. Following Myerson (1982) and the discussion in Sect. 1.4, we allow player 0:

- as auctioneer, to announce the Walrasian equilibrium price vector $\bar{p}$;

- as principal, to recommend that each trader $i \in I$ chooses any specific selection $p \mapsto z^{i}(p)$ from the Walrasian net demand correspondence (10) that satisfies $z^{i}(\bar{p})=\bar{z}^{i}$ at the chosen equilibrium price vector.

This selects a particular subgame perfect Nash equilibrium which does implement the chosen Walrasian equilibrium $\left(\bar{p}, \bar{z}^{I}\right)$.

\subsection{Untrustworthy traders}

The game defined in Sect. 3.1, however, makes the outcome function depend only on the traders' profile of net trade vectors $\bar{z}^{I}$ at the equilibrium price vector $\bar{p} \in P^{0}$. These net trades are based on the promise and the premise that each trader will supply the equilibrium supply vector $\bar{y}^{i}=-\left(\bar{z}^{i} \wedge 0\right)$. Yet no clear incentive for them to do so has been provided.

Indeed, unlike Shapley and Shubik (1977), as well as many successors, the game defined in Sect. 3.1 ignores what commodity vectors traders actually choose to supply. To overcome this moral hazard problem, we construct an alternative multistage game that incorporates a warehouse system.

\section{A game with warehousing}

\subsection{Distinguishing supply and demand}

Our mechanism with warehousing takes place in several stages. It begins very like the reformulated Arrow-Debreu game of Sect. 3.1. The difference is that we distinguish between supply and demand and require each trader $i \in I$ to choose what supply vector $y^{i}$ to deposit in a warehouse system. Only at a later stage is each trader $i \in I$ allowed to withdraw a demand vector $x^{i}$ from the warehouse system.

We therefore consider the multistage game that starts as follows:

1. Initially, the auctioneer selects some Walrasian equilibrium price vector $\bar{p} \in P^{0}$ and also recommends an associated mandated Walrasian equilibrium allocation $\bar{z}^{I}$ of net trade vectors, with associated profiles $\bar{x}^{I}, \bar{y}^{I} \in\left(\mathbb{R}_{+}^{G}\right)^{I}$ of non-negative mandated warehouse demand vectors $\bar{x}^{i}:=\bar{z}^{i} \vee 0$ and supply vectors $\bar{y}^{i}=$ $-\left(\bar{z}^{i} \wedge 0\right)=\left(-\bar{z}^{i}\right) \vee 0$ defined for all $i \in I$ following Eq. (3). Note that because $e^{i} \geqq 0$, the feasibility constraint $\bar{z}^{i} \geqq-e^{i}$ implies that $\bar{y}^{i} \leqq e^{i}$.

2. Next, each trader $i \in I$ chooses to deposit in the warehouse system some supply vector $y^{i} \in R_{+}^{G}$. Because the warehouse manager is told only to accept the supply of any good up to the limit of what is mandated, this supply vector is required to satisfy $y^{i} \leqq \bar{y}^{i}$. 


\subsection{Distinguishing deviant from compliant agents}

The moves made in these first two stages of the game determine the two profiles $\bar{y}^{I}$ and $y^{I}$ of mandated and actual warehouse deposit vectors, respectively. These profiles also determine a partition of $I$ into two disjoint subsets of traders:

- the set

$$
C\left(y^{I}, \bar{y}^{I}\right):=\left\{i \in I \mid y^{i}=\bar{y}^{i}\right\}
$$

of compliant traders, who deliver their mandated supplies, and will be duly rewarded;

- the complementary set

$$
D\left(y^{I}, \bar{y}^{I}\right):=I \backslash C\left(y^{I}, \bar{y}^{I}\right)=\left\{i \in I \mid y^{i}<\bar{y}^{i}\right\}
$$

of deviant traders, who fail to deliver their mandated supplies, and will be banished entirely from the warehouse system.

\subsection{The normal ending of the game}

Suppose that the two supply profiles $y^{I}, \bar{y}^{I} \in\left(\mathbb{R}_{+}^{G}\right)^{I}$ of different traders' actual and mandated warehouse supply vectors are equal. Then $C\left(y^{I}, \bar{y}^{I}\right)=I$. In this case, each trader $i$ is allowed to withdraw any commodity vector $x^{i} \in \mathbb{R}_{+}^{G}$ satisfying $x^{i} \leqq \bar{x}^{i}:=\bar{z}^{i} \vee 0$. After these withdrawals have been completed, any remaining surplus is thrown away. This is the end of the game.

\subsection{The abnormal ending of the game}

Alternatively, suppose that for the pair $\left(y^{I}, \bar{y}^{I}\right)$ of warehouse deposit profiles, there exists $i \in I$ such that $y^{i}<\bar{y}^{i}$-or equivalently, suppose that the set $D\left(y^{I}, \bar{y}^{I}\right)$ of deviant traders is non-empty. In this case, a second-period allocation submarket is opened, whose outcome depends on $\left(y^{I}, \bar{y}^{I}\right)$. In this submarket, the auctioneer will choose a revised price vector $q \in P^{0}$ to clear markets given the already determined available aggregate supply vector $\sum_{i \in I} y^{i}$. Moreover, each trader $i \in I$ is faced with a second-period budget constraint $q x^{i} \leq w^{i}\left(q, y^{I}, \bar{y}^{I}\right)$, whose right-hand side is determined by a continuous wealth distribution rule $q \mapsto w^{i}\left(q, y^{I}, \bar{y}^{I}\right)$ which also depends on the mandated as well as the actual warehouse deposit vectors.

Specifically, for each pair $y^{I}, \bar{y}^{I} \in\left(\mathbb{R}_{+}^{G}\right)^{I}$, the market organizer:

1. first specifies a continuous wealth distribution rule (or WDR)

$$
P^{0} \ni q \mapsto w^{I}\left(q, y^{I}, \bar{y}^{I}\right) \in \mathbb{R}^{I}
$$

as a function of the price vector $q$ that is about to be chosen;

2. given this WDR, specifies some Walrasian equilibrium selection rule

$$
\mathbb{R}^{G} \times \mathbb{R}^{G} \ni\left(y^{I}, \bar{y}^{I}\right) \mapsto\left(\tilde{q}, \tilde{x}^{I}\right)\left(y^{I}, \bar{y}^{I}\right) \in P^{0} \times\left(\mathbb{R}_{+}^{G}\right)^{I}
$$


which generates net demand vectors that, for each $y^{I}, \bar{y}^{I} \in\left(\mathbb{R}_{+}^{G}\right)^{I}$, satisfy the market clearing condition

$$
\sum_{i \in I} \tilde{x}^{i}\left(y^{I}, \bar{y}^{I}\right)=\sum_{i \in I} y^{i}
$$

3. allows each trader $i$ to withdraw any demand vector $x^{i} \in \mathbb{R}_{+}^{G}$ satisfying $x^{i} \leqq$ $\tilde{x}^{i}\left(y^{I}, \bar{y}^{I}\right)$, and then ends the game by throwing away any outstanding stocks.

\subsection{The second-period wealth distribution rule}

Banishing any deviant trader $i \in D\left(y^{I}, \bar{y}^{I}\right)$ is equivalent to leaving them with nothing at all to spend in the second-period market, so

$$
w^{i}\left(q, y^{I}, \bar{y}^{I}\right):=0 \quad \text { if } i \in D\left(y^{I}, \bar{y}^{I}\right)
$$

This draconian sanction is imposed in order to ensure that the income loss outweighs anything that a deviating trader could possibly gain by manipulating warehouse supplies of one or more goods in an attempt to force the auctioneer to change prices in a favourable direction. ${ }^{4}$

Compared to allowing all deviant traders $i \in D\left(y^{I}, \bar{y}^{I}\right)$ to spend the market value $q y^{i}$ of what they have actually delivered, this sanction generates a positive total surplus given by

$$
S\left(q, y^{I}, \bar{y}^{I}\right):=\sum_{i \in D\left(y^{I}, \bar{y}^{I}\right)} q y^{i}
$$

This surplus will then be shared equally between all compliant traders $i \in C\left(y^{I}, \bar{y}^{I}\right)$. It will supplement the value $q \bar{y}^{i}$ at prices $q$ of trader $i$ 's mandated deliveries to the warehouse system. This implies that any compliant trader $i \in C\left(y^{I}, \bar{y}^{I}\right)$, for whom $y^{i}=\bar{y}^{i}$ by definition, is allowed to spend the amount

$$
w^{i}\left(q, y^{I}, \bar{y}^{I}\right):=q y^{i}+\frac{1}{\# C\left(y^{I}, \bar{y}^{I}\right)} \sum_{h \in D\left(y^{I}, \bar{y}^{I}\right)} q y^{h}
$$

Overall, therefore, we define the second-period WDR by

$$
w^{i}\left(q, y^{I}, \bar{y}^{I}\right):= \begin{cases}q y^{i}+\frac{1}{\# C\left(y^{I}, \bar{y}^{I}\right)} \sum_{h \in D\left(y^{I}, \bar{y}^{I}\right)} q y^{h} & \text { if } y^{i}=\bar{y}^{i} \\ 0 & \text { if } y^{i}<\bar{y}^{i}\end{cases}
$$

Note that if $y^{i}=\bar{y}^{i}$, then $\# C\left(y^{I}, \bar{y}^{I}\right) \geq 1$, so $w^{i}\left(q, y^{I}, \bar{y}^{I}\right)$ is always well defined. Summing equations (17) over $i \in I$ shows that this WDR satisfies

\footnotetext{
4 Many thanks to John Geanakoplos for emphasizing the importance of this point.
} 


$$
\sum_{i \in I} w^{i}\left(q, y^{I}, \bar{y}^{I}\right)=\sum_{i \in C\left(y^{I}, \bar{y}^{I}\right)} w^{i}\left(q, y^{I}, \bar{y}^{I}\right) \leq \sum_{i \in I} q y^{i}
$$

with equality except in the degenerate case when $C\left(y^{I}, \bar{y}^{I}\right)=\emptyset$ and so $w^{i}\left(q, y^{I}, \bar{y}^{I}\right)=$ 0 for all $i \in I$. In this degenerate case, all traders are excluded from the warehouse, whose entire contents are then discarded. In the non-degenerate case, reaching an equilibrium allocation in the second-period market system will allow the warehouse to be emptied.

\subsection{Second-period Walrasian equilibrium}

For each fixed pair $\left(y^{I}, \bar{y}^{I}\right) \in\left(\mathbb{R}_{+}^{G}\right)^{I} \times\left(\mathbb{R}_{+}^{G}\right)^{I}$, the mapping $q \mapsto w^{i}\left(q, y^{I}, \bar{y}^{I}\right)$ whose value is defined by (17) is always continuous in the price vector $q$. So a variation of the usual existence argument shows that there is a Walrasian equilibrium in this secondperiod submarket. We assume that the auctioneer/principal uses an arbitrary selection rule to choose a mapping

$$
\left(y^{I}, \bar{y}^{I}\right) \mapsto\left(q\left(y^{I}, \bar{y}^{I}\right), \tilde{x}^{I}\left(y^{I}, \bar{y}^{I}\right)\right)
$$

whose value is always a Walrasian equilibrium with an allocation $\tilde{x}^{I}\left(y^{I}, \bar{y}^{I}\right)$ of warehouse withdrawal vectors that satisfies the market clearing condition (15).

For notational simplicity, we extend the domain of the mapping defined by (19) to include pairs of profiles $\left(y^{I}, \bar{y}^{I}\right) \in\left(\mathbb{R}_{+}^{G}\right)^{I} \times\left(\mathbb{R}_{+}^{G}\right)^{I}$ satisfying $y^{I}=\bar{y}^{I}$ or equivalently $C\left(y^{I}, \bar{y}^{I}\right)=I$. Note that then Eq. (17) specifies that the second-period WDR must satisfy

$$
\sum_{i \in I} w^{i}\left(q, y^{I}, \bar{y}^{I}\right)=q \sum_{i \in I} y^{i}=q \sum_{i \in I} \bar{y}^{i}=q \sum_{i \in I} \bar{x}^{i}
$$

This allows us to specify that the value of the mapping (19) at $\left(y^{I}, \bar{y}^{I}\right)$ should be given by $q\left(y^{I}, \bar{y}^{I}\right)=\bar{p}$ and $\tilde{x}^{I}\left(y^{I}, \bar{y}^{I}\right)=\bar{x}^{I}$.

\subsection{A second-period mechanism}

To convert this second-period submarket, with its selected Walrasian equilibrium, into a strategic mechanism, with its corresponding Nash equilibrium, we modify the firstperiod mechanism of Sect. 4.1. Specifically, following each pair of profiles $\left(y^{I}, \bar{y}^{I}\right) \in$ $\left(\mathbb{R}_{+}^{G}\right)^{I} \times\left(\mathbb{R}_{+}^{G}\right)^{I}:$

1. At the start of the second period, the market organizer applies the selection rule (19) in order to determine a Walrasian equilibrium with price vector $\tilde{q}\left(y^{I}, \bar{y}^{I}\right) \in P^{0}$ and associated demand allocation $\tilde{x}^{I}\left(y^{I}, \bar{y}^{I}\right)$.

2. Subsequently, each trader $i \in I$ withdraws from the warehouse a commodity vector $x^{i} \in \mathbb{R}_{+}^{G}$ satisfying $x^{i} \leqq \tilde{x}^{i}\left(y^{I}, \bar{y}^{I}\right)$; the game ceases when these withdrawals have been completed and any remaining stocks thrown away. 
Because preferences are assumed to be strictly monotone, these rules evidently imply that withdrawing the full allowance $\tilde{x}^{i}\left(y^{I}, \bar{y}^{I}\right)$ is a strictly dominant strategy for each trader $i \in I$ in the subgame that follows the principal's choice $\left(\tilde{q}\left(y^{I}, \bar{y}^{I}\right), \tilde{x}^{I}\left(y^{I}, \bar{y}^{I}\right)\right.$ of Walrasian equilibrium.

\subsection{First-period pay-offs and dominant strategies}

Looking back to the first period, recall our postulate that the auctioneer/principal chooses a particular Walrasian equilibrium

$$
\left(\bar{p}, \bar{x}^{I}, \bar{y}^{I}\right) \in P^{0} \times\left(\mathbb{R}_{+}^{G}\right)^{I} \times\left(\mathbb{R}_{+}^{G}\right)^{I}
$$

for the exchange economy. After the auctioneer/principal has made this choice, consider the pay-off function $y^{I} \mapsto v^{i}\left(y^{I}\right)$ of each trader $i \in I$ in the ensuing first-period subgame where:

1. each player $i \in I$ has a strategy space consisting of warehouse deposit vectors satisfying $0 \leqq y^{i} \leqq \bar{y}^{i}$;

2. thereafter the economy continues on to the second-period Walrasian equilibrium $q\left(y^{I}, \bar{y}^{I}\right), \tilde{x}^{I}\left(y^{I}, \bar{y}^{I}\right)$ specified by (19) as the outcome of the ensuing secondperiod subgame.

The rules devised in Sects. 4.4 and 4.5 to determine this second-period Walrasian equilibrium imply that this pay-off function satisfies

$$
v^{i}\left(y^{I}\right)= \begin{cases}u^{i}\left(e^{i}-y^{i}+\tilde{x}^{i}\left(y^{I}, \bar{y}^{I}\right)\right) & \text { if } y^{i}=\bar{y}^{i} \\ u^{i}\left(e^{i}-y^{i}\right) & \text { if } y^{i} \neq \bar{y}^{i}\end{cases}
$$

with $\tilde{x}^{i}\left(y^{I}, \bar{y}^{I}\right)=\bar{x}^{i}$ in case $y^{I}=\bar{y}^{I}$. Because preferences are strictly increasing, while both $\bar{x}^{i}$ and $\tilde{x}^{i}\left(y^{I}, \bar{y}^{I}\right)$ are non-negative, it follows that whenever $y^{i}<\bar{y}^{i}$ one has

$$
v^{i}\left(y^{I}\right)=u^{i}\left(e^{i}-y^{i}\right) \leq u^{i}\left(e^{i}-y^{i}+\tilde{x}^{i}\left(y^{I}, \bar{y}^{I}\right)\right)=v^{i}\left(\bar{y}^{i}, y^{I \backslash\{i\}}\right)
$$

with strict inequality except in the special case when $\tilde{x}^{i}\left(\bar{y}^{i}, y^{I \backslash\{i\}}, \bar{y}^{I}\right)=0$. Hence, the minimal compliant supply strategy $y^{i}=\bar{y}^{i}$ is player $i$ 's unique best response in this first-period game to any strategy profile $y^{I \backslash\{i\}}$ chosen by the other players.

\subsection{Subgame perfect dominant strategies}

Consider the two-stage strategic market game defined in this section. Suppose that the auctioneer/principal is committed to choose both:

1. the first-stage mandated Walrasian equilibrium $\left(\bar{p}, \bar{x}^{I}, \bar{y}^{I}\right)$, as described in Sect. 4.1;

2. for each profile $y^{I}$ of actual warehouse supply vectors, and given the profile $\bar{y}^{I}$ of mandated warehouse supply vectors that was determined in the first period, the second-stage Walrasian equilibrium $\left(q, \tilde{x}^{I}\right)\left(y^{I}, \bar{y}^{I}\right)$, as described in Sect. 4.6. 
Conditional on these commitments, all the traders are involved in a two-stage game where each trader $i \in I$ is required to choose:

1. in the first stage, a non-negative warehouse deposit vector satisfying $y^{i} \leqq \bar{y}^{i}$;

2. in the second stage, in each possible subgame determined by a specific pair $\left(y^{I}, \bar{y}^{I}\right)$ of actual and mandated warehouse supply vectors, a non-negative warehouse withdrawal vector satisfying $x^{i} \leqq \tilde{x}^{i}\left(y^{I}, \bar{y}^{I}\right)$.

Given the assumption that preferences are strictly monotone, our construction ensures that each trader $i \in I$ has:

1. in the overall two-stage game, a strictly dominant strategy consisting of:

- the minimal compliant supply strategy in the first stage of depositing the mandated amount $\bar{y}^{i}$ into the warehouse system, as described in Sect. 4.8;

- followed in every possible second-stage subgame defined by the pair $\left(y^{I}, \bar{y}^{I}\right)$ by the maximal demand strategy of withdrawing the full amount $\tilde{x}^{i}\left(y^{I}, \bar{y}^{I}\right)$ allowed to trader $i$.

2. within every possible second-stage subgame defined by the pair $\left(y^{I}, \bar{y}^{I}\right)$, a strictly dominant strategy consisting of the same maximal demand strategy.

In this sense, the strategy profile where every trader pursues this combination of the minimal compliant supply strategy followed by the maximal demand strategy is a subgame perfect Nash equilibrium in subgame perfect strictly dominant strategies. Of course, it does implement the Walrasian equilibrium chosen by the market principal.

\section{An economy with many traders}

\subsection{Incomplete information}

In the game set out in Sect. 3, player 0 as market manager combined the role of the pricesetting Walrasian auctioneer with that of the principal in a generalized principal/agent model. We now switch focus to the case when player 0 has incomplete information regarding traders' preferences and endowments.

In Sect. 4, the same player 0 also took on the role of warehouse manager, but with complete information regarding the traders. When there is incomplete information, we defer until Sect. 7 any discussion of the incentives needed to encourage traders to deposit their promised supply vectors in a warehouse.

\subsection{Traders and their labels}

We will be considering strategyproof trading mechanisms in a setting with incomplete information. The introduction explained why we need to consider an economy with many traders. Accordingly, let the set $I$ of actual traders be $\mathbb{N}:=\{1,2, \ldots\}$, the countably infinite set of natural numbers. There will, however, be a continuum of potential traders in the form of a non-atomic probability space $(L, \mathscr{L}, \lambda)$, where $L$ is a topological label space, with $\mathscr{L}$ as its completed Borel $\sigma$-algebra-i.e. it contains not only all open sets, but all subsets of $\lambda$-null sets. A prominent example occurs 
when $L$ is the unit interval $[0,1]$ equipped with its Lebesgue $\sigma$-algebra $\mathscr{L}$ and its Lebesgue measure $\lambda$.

Now each trader $i \in I$ will be given a random label $\ell^{i}$ chosen from a non-atomic probability space $(L, \mathscr{L}, \lambda)$. This random labelling embeds an economy with a countable set of traders into the standard notion of a continuum economy with $L$ as the set of potential traders. In this continuum economy, there is an actual trader labelled $\ell$ if and only if the label $\ell$ happens to equal one of the countable random draws from $(L, \mathscr{L}, \lambda)$.

\subsection{Traders' types}

To accommodate incomplete information, we follow Harsanyi (1967-1968) in assuming that traders' pay-off functions depend on their respective hidden personal types, known only to themselves. Actually, unlike in Harsanyi's formulation, in Sects. 7 and 8 traders will also have hidden endowments, and so feasible sets of net trade vectors that depend on hidden variables.

Specifically, assume first that each trader $i$ 's utility function $\mathbb{R}_{+}^{G} \ni c^{i} \mapsto u^{i}\left(c^{i}\right) \in \mathbb{R}$, which was introduced in Sect. 2.5, takes the form $u^{i}\left(c^{i}\right) \equiv u\left(c^{i} ; \theta^{i}\right)$ for a taste parameter $\theta^{i}$ belonging to a type space $\Theta$, where the mapping

$$
\mathbb{R}_{+}^{G} \times \Theta \ni(c, \theta) \mapsto u(c ; \theta) \in \mathbb{R}
$$

is independent of $i$, as well as jointly continuous and strictly increasing in $c$ for each fixed $\theta$. Quasi-concavity, however, will not be required for existence of Walrasian equilibrium in our economy with an infinite set of traders.

Second, define the rectangular set $\bar{E}=\left\{e \in \mathbb{R}_{+}^{G} \mid e \leqq \bar{e}\right\}$ for a suitable upper bound satisfying $\bar{e} \gg 0$. This strict inequality loses no generality provided that we exclude from the commodity set $G$ those goods $g$ for which $\bar{e}_{g}=0$.

We will take the space of possible endowment vectors $e$ to be the set

$$
E:=\bar{E} \backslash\{0\}=\left\{e \in \mathbb{R}_{+}^{G} \mid 0<e \leqq \bar{e}\right\}
$$

This implicitly excludes any trader whose endowment vector is zero and who therefore plays no role in any Walrasian exchange.

Finally, the traders' type space is taken to be the Cartesian product space $T=\Theta \times E$, whose members are hidden preference-endowment pairs $t=(\theta, e)$.

\subsection{A statistical continuum economy}

Let $\mathscr{M}_{\lambda}(L ; T)$ denote the family of probability measures $\tau$ on the product measurable space $(L \times T, \mathscr{B}(L \times T))$ of label-type pairs with the property that the marginal measure induced by $\tau$ on the component space $L$ equals the specified measure $\lambda$. That is, for every Borel subset $B$ in $L$, one has $\tau(B \times T)=\lambda(B)$. A statistical continuum economy is defined as a probability measure or type distribution $\tau \in \mathscr{M}_{\lambda}(L ; T)$. 
Given any Borel set $B \subseteq L \times T$ and any label $\ell \in L$, define the section $B_{\ell}:=$ $\{t \in T \mid(\ell, t) \in B\}$ of $B$. Then, as discussed in Appendix 1, the requirement that $\operatorname{marg}_{L} \tau=\lambda$ is equivalent to the existence of a stochastic transition or measure disintegration in the form of a measurable mapping $L \ni \ell \mapsto \tau_{\ell} \in \mathscr{M}(T)$ which is also the regular conditional probability measure that satisfies $\tau(B)=\int_{L} \tau_{\ell}\left(B_{\ell}\right) \lambda(\mathrm{d} \ell)$ for every Borel set $B \subseteq L \times T$. In Appendix 1, it is also shown that, because $\lambda$ is non-atomic, so is $\tau$.

Thus, in the end our economy will have the countable set $I=\mathbb{N}$ of actual traders, whose labels $\left(\ell^{i}\right)_{i \in \mathbb{N}}$ are assumed to be i.i.d. random variables drawn from the probability space $(L, \mathscr{L}, \lambda)$. Moreover, it is assumed that there exists a probability measure $\tau \in \mathscr{M}_{\lambda}(L ; T)$ such that the actual traders' label-type pairs $\left(\ell^{i}, t^{i}\right)_{i \in \mathbb{N}}$ are independent and identically distributed (i.i.d.) random variables drawn from the product probability space $(L \times T, \mathscr{B}(L \times T), \tau)$. Then, each label-type pair $(\ell, t) \in L \times T$ can be regarded as a potential trader, whose existence as an actual trader depends on whether $(\ell, t)$ is included in the set $\left\{\left(\ell^{i}, t^{i}\right) \mid i \in \mathbb{N}\right\}$ of random draws. Because $\tau$ is non-atomic, the probability space $(L \times T, \mathscr{B}(L \times T), \tau)$ can be regarded as a continuum of potential traders.

\subsection{The statistical economy as limit}

Given any label-type pair $(\ell, t) \in L \times T$, let $\delta_{(\ell, t)}$ denote the degenerate Borel probability measure defined on the space $\mathscr{B}(L \times T)$ of Borel measurable subsets of $L \times T$ which, for every Borel set $B \subset L \times T$, satisfies

$$
\delta_{(\ell, t)}(B):= \begin{cases}1, & \text { if }(\ell, t) \in B \\ 0, & \text { if }(\ell, t) \notin B\end{cases}
$$

For each infinite sequence $\left(\ell^{\mathbb{N}}, t^{\mathbb{N}}\right)=\left(\ell^{k}, t^{k}\right)_{k \in \mathbb{N}}$ of label-type pairs in $L \times T$, and for each $n \in \mathbb{N}$, let

$$
\tau_{n}\left(\ell^{\mathbb{N}}, t^{\mathbb{N}}\right):=\frac{1}{n} \sum_{k=1}^{n} \delta_{\left(\ell^{k}, \theta^{k}\right)}
$$

denote the empirical measure on Borel subsets of $L \times T$ generated by the label-type pairs of the finite subset of traders $\{1,2, \ldots, n\}$. That is, by (26) and (27), the empirical measure of each Borel set $B \subset L \times T$ satisfies

$$
\tau_{n}\left(\ell^{\mathbb{N}}, t^{\mathbb{N}}\right)(B)=\frac{1}{n} \sum_{k=1}^{n} \delta_{\left(\ell^{k}, \theta^{k}\right)}(B)=\frac{1}{n} \#\left\{k \in\{1,2, \ldots, n\} \mid\left(\ell^{k}, \theta^{k}\right) \in B\right\}
$$

This requires $\tau_{n}\left(\ell^{\mathbb{N}}, t^{\mathbb{N}}\right)(B)$ to equal the proportion of the set of $n$ pairs $\left\{\left(\ell^{k}, t^{k}\right) \mid k \in\right.$ $\{1,2, \ldots, n\}\}$ that belong to $B$.

Because both $L$ and $T$ are compact Polish spaces, so is $L \times T$ given any natural metric. Equip the space $\mathscr{M}(L \times T)$ of joint probability measures with its topology of weak convergence of probability measures, along with the associated Borel $\sigma$-algebra; 
this makes $\mathscr{M}(L \times T)$ a compact Polish space as well. Recall the assumption that the sequence $\left(\ell^{\mathbb{N}}, t^{\mathbb{N}}\right)=\left(\ell^{k}, t^{k}\right)_{k \in \mathbb{N}}$ of label-type pairs results from infinitely many i.i.d. random draws from the common probability space $(L \times T, \mathscr{B}(L \times T), \tau)$. As discussed in Appendix 1, this assumption enables the Glivenko-Cantelli theorem to be applied; it tells us that for $\tau^{\infty}$-a.e. sequence $\left(\ell^{\mathbb{N}}, t^{\mathbb{N}}\right)$, in this weak convergence topology the empirical measure $\tau_{n} \rightarrow \tau$ as $n \rightarrow \infty$.

Moreover, definition (27) implies that the marginal empirical measure over the measurable label space $(L, \mathscr{L})$ satisfies

$$
\operatorname{marg}_{L} \tau_{n}=\frac{1}{n} \sum_{k=1}^{n} \operatorname{marg}_{L} \delta_{\left(\ell^{k}, \theta^{k}\right)}=\frac{1}{n} \sum_{k=1}^{n} \delta_{\ell^{k}}
$$

Applying the Glivenko-Cantelli theorem to the left-hand side of (29) establishes that $\operatorname{marg}_{L} \tau_{n} \rightarrow \operatorname{marg}_{L} \tau$ for $\lambda^{\infty}$-a.e. sequence $\ell^{\mathbb{N}}$, whereas applying it to the right-hand side establishes that $\frac{1}{n} \sum_{k=1}^{n} \delta_{\ell^{k}} \rightarrow \lambda$ for $\lambda^{\infty}$-a.e. sequence $\ell^{\mathbb{N}}$. So these limits must be equal, from which it follows that $\operatorname{marg}_{L} \tau=\lambda$ and so $\tau \in \mathscr{M}_{\lambda}(L ; T)$.

This convergence property enables us to interpret the statistical continuum economy with a continuum of potential traders $(\ell, t) \in L \times T$ as an appropriate limit of an economy with a countable set of traders $k \in \mathbb{N}$ whose label-type pairs $\left(\ell^{k}, t^{k}\right)_{k \in \mathbb{N}}$ are i.i.d. random draws from a joint probability measure $\tau \in \mathscr{M}_{\lambda}(L ; T)$ over $L \times T$ whose marginal on $L$ is $\lambda$.

\subsection{Statistical strategy measures}

Consider a trading game of incomplete information in which a market organizer confronts a finite set $I$ of traders. Each trader chooses a strategy belonging to a common strategy or action set $A$ that takes the form of a Polish space. Then it is natural to consider the entire domain of strategy profiles

$$
I \times T \ni(i, t)=(i, \theta, e) \mapsto a^{i, t} \in A
$$

determining, as a function of their respective individual type $t \in T$, the strategy of each trader $i \in I$.

We focus on the extension to a countably infinite set of traders whose label-type pairs form the sequence $\left(\ell^{\mathbb{N}}, t^{\mathbb{N}}\right)$ of independent random draws from the probability measure $\tau$ over Borel subsets of $L \times T$. In this case, it seems natural at first to extend this notion of strategy profile to mappings

$$
L \times T \ni(\ell, t)=(\ell, \theta, e) \mapsto a^{\ell, t} \in A
$$

Yet the space $A^{L \times T}$ of all these mapping includes many that are not measurable w.r.t. any convenient $\sigma$-algebra on $L \times T$. Instead of mappings in $A^{L \times T}$, therefore, we focus on statistics that the market organizer could estimate, at least in principle.

Indeed, suppose first that the market organizer was able to observe not only the traders' labels $\ell \in L$ and actions $a \in A$, but also their hidden types $t \in T$. Then 
it is assumed that the market organizer's estimated distribution of triples $(\ell, t, a) \in$ $L \times T \times A$ takes the form of a statistical strategy measure $\alpha^{S}$ in the space $\mathscr{M}_{\tau}(L \times T ; A)$ of joint probability measures on $L \times T \times A$ whose marginal distribution $\operatorname{marg}_{L \times T} \alpha^{S}$ on the component subspace $L \times T$ of label-type pairs equals the exogenously specified joint measure $\tau \in \mathscr{M}_{\lambda}(L ; T)$. In effect, we assume that:

1. not only does the infinite sequence $\left(\ell^{\mathbb{N}}, t^{\mathbb{N}}\right) \in(L \times T)^{\mathbb{N}}$ of actual traders' labeltype pairs consist of i.i.d. random draws from a joint probability measure $\tau \in$ $\mathscr{M}_{\lambda}(L ; T)$ over potential traders' label-type pairs;

2. but there also exists a joint probability measure $\alpha^{S} \in \mathscr{M}_{\tau}(L \times T ; A)$ over potential traders' triples $(\ell, t, a)$ having the property that, when the actual traders' observable actions $a^{k} \in A$ are appended to their label-type pairs $\left(\ell^{k}, t^{k}\right)$, the resulting infinite sequence $\left(\ell^{\mathbb{N}}, t^{\mathbb{N}}, a^{\mathbb{N}}\right)$ of triples $\left(\ell^{k}, t^{k}, a^{k}\right)$ consists of i.i.d. random draws from the distribution $\alpha^{S}$ over $L \times T \times A$.

An unnecessarily strong sufficient condition for the measure $\alpha^{S}$ to exist can be specified, following the results of Hammond and Sun (2008). It requires that there exist:

1. a probability space $(\Omega, \mathscr{F}, P)$;

2. a measurable mapping $L \times T \ni(\ell, t) \mapsto \pi^{\ell, t} \in \mathscr{M}(A)$ from the space of labeltype pairs $(\ell, t)$ to the domain of probability measures, or equivalently of mixed strategies, on $A$;

3. a random process

$$
L \times T \times \Omega \ni(\ell, t, \omega) \mapsto \alpha^{\ell, t}(\omega) \in A
$$

in which the random variables $\Omega \ni \omega \mapsto \alpha^{\ell, t}(\omega)$ are independent, like independently mixed strategies, with respective marginal distributions $\pi^{\ell, t}$.

Under this assumption, the measure $\alpha^{S}$ not only describes the joint distribution of the the continuum of potential agents' triples $(\ell, t, a) \in L \times T \times A$. Relative to the product probability measure $\left(\alpha^{S}\right)^{\mathbb{N}}$ on the Borel product $\sigma$-algebra of the infinite Cartesian product space $(L \times T \times A)^{\mathbb{N}}$ of triple sequences $\left(\ell^{\mathbb{N}}, t^{\mathbb{N}}, a^{\mathbb{N}}\right)$, it almost surely also describes the distribution of the list of actual agents' triples.

The market organizer, however, cannot observe any trader's type. So we assume that its decisions are based on the estimated marginal distribution $\alpha=\operatorname{marg}_{L \times A} \alpha^{S} \in$ $\mathscr{M}_{\lambda}(L ; A)$ of observable label-action pairs $(\ell, a) \in L \times A$.

A similar formulation will appear repeatedly in the subsequent analysis.

\section{A demand revelation mechanism}

\subsection{The exchangeable commodity set}

In our demand revelation (or DR) game, there will be one market organizer who confronts a countably infinite set of agents whose label-type pairs $\left(\ell^{k}, t^{k}\right)_{k \in \mathbb{N}}$ are i.i.d. random draws from the non-atomic probability measure $\tau \in \mathscr{M}_{\lambda}(L ; T)$ over the Borel subsets of the Cartesian product set $L \times T$ of potential agents' label-type pairs. 
For demand revelation to succeed in reaching a Walrasian equilibrium, that equilibrium must exist. To ensure that it does, it is important to avoid the difficulty created by Arrow's (1951) exceptional case, especially in the later versions considered by Koopmans (1957) and many others, including Hammond (2011). This difficulty can be attributed to the misguided attempt to create a market for a good $g \in G$ even when no trader has any endowment of $g$, so $g$ can never be traded.

Accordingly, the demand revelation game will start by having each actual trader with the label-type pair $(\ell, t) \in L \times T$ announce an endowment set $H^{\ell, t} \subseteq G$ of commodities that the trader claims to be able to supply in positive amounts. All the actual traders' announcements are assumed to give rise to an estimated statistical strategy measure $\gamma \in \mathscr{M}_{\lambda}\left(L ; 2^{G}\right)$ over observable pairs $(\ell, H) \in L \times 2^{G}$ of potential agents' labels and associated endowment sets, where $2^{G}$ denotes the power set of all subsets of the finite set $G$ of potentially exchangeable commodities.

For each $g \in G$, define the set

$$
2_{g}^{G}:=\left\{H \in 2^{G} \mid g \in H\right\}
$$

of subsets of $G$ that contain the particular good $g$. Based on each possible estimated measure $\gamma$, let

$$
G(\gamma):=\left\{g \in G \mid \gamma\left(L \times\left\{H \in 2^{G} \mid g \in H\right\}\right)>0\right\}
$$

denote the trading set of those commodities which a non-null set of potential traders have included in their announced endowment set. Often this set will be denoted simply by $K$. In case $\# K<2$, essentially no trade is possible; so we assume from now on that $\#=2$.

The demand revelation mechanism we construct will ignore all essentially nonexchangeable commodities outside the set $K:=G(\gamma)$. Accordingly, given $K$, define the trading space $\mathbb{R}^{K}$, as well as the new price simplex

$$
P_{K}:=\left\{p \in \mathbb{R}_{+}^{K} \mid \sum_{g \in G(\gamma)} p_{g}=1\right\}
$$

with relative interior

$$
P_{K}^{0}:=\left\{p \in \mathbb{R}_{++}^{K} \mid \sum_{g \in G(\gamma)} p_{g}=1\right\}
$$

and relative boundary bd $P_{K}:=P_{K} \backslash P_{K}^{0}$.

\subsection{Regular demands}

Once the set $K:=G(\gamma)$ of exchangeable commodities has been determined, the budget correspondence of any trader is defined by the mapping 


$$
P_{K}^{0} \ni p \mapsto B^{K}(p):=\left\{z \in \mathbb{R}^{K} \mid p z \leq 0\right\}
$$

Then a possible net demand correspondence will be a mapping

$$
P_{K}^{0} \ni p \mapsto Z(p ; \mathfrak{Z}) \subset B^{K}(p)
$$

also denoted by $\mathfrak{Z}$, that selects a non-empty subset $Z(p ; \mathfrak{Z})$ of the budget set $B^{K}(p)$ for each exchangeable goods price vector $p \in P_{K}^{0}$.

Given the trading set $K:=G(\gamma)$, we may as well replace each trader's announced endowment set $H \subset G$ by the effective endowment set defined as the intersection $J:=H \cap K$.

Recall that in (25) of Sect. 5.3 we introduced the notation $\bar{e} \in \mathbb{R}_{++}^{G}$ for the uniform upper bound on traders' endowment vectors. Given any announced effective endowment set $J \subseteq K$, define $\bar{e}^{J}:=(\bar{e})_{g \in J} \in \mathbb{R}_{+}^{J}$ as the subvector of $\bar{e}$ that results from considering only goods $g \in J$, and $\left(\bar{e}^{J}, 0^{K \backslash J}\right) \in \mathbb{R}_{+}^{K}$ as the vector that results after replacing by zero all the components $\left(\bar{e}_{g}\right)_{g \in K \backslash J}$ corresponding to the other goods. Similarly, for each price vector $p=\left(p_{g}\right)_{g \in K} \in P_{K}$, one can define the vector $p^{J}=\left(p_{g}\right)_{g \in J}$ of associated components.

Definition 1 Given the trading set $K$ and the announced endowment set $H \subseteq G$ whose intersection with $K$ is $J$, a net demand correspondence $\mathfrak{Z}$ given by (34) is said to be $J$-regular just in case it satisfies the following four conditions:

1. budget exhaustion: $p z=0$ for all $p \in P_{K}^{0}$ and all $z \in Z(p ; \mathfrak{Z})$;

2. feasibility: $z \geqq-\left(\bar{e}^{J}, 0^{K \backslash J}\right)$ for all $p \in P_{K}^{0}$ and for all $z \in Z(p ; \mathfrak{Z})$;

3. continuity: the correspondence $\mathfrak{Z}$ has a graph

$$
\Gamma_{\mathfrak{Z}}:=\left\{(p, z) \in P_{K}^{0} \times \mathbb{R}^{K} \mid z \in Z(p ; \mathfrak{Z})\right\}
$$

which is a relatively closed subset of $P_{K}^{0} \times \mathbb{R}^{K}$;

4. boundary condition: Suppose that $\left(p^{\mathbb{N}}, z^{\mathbb{N}}\right)=\left(p^{n}, z^{n}\right)_{n \in \mathbb{N}} \in P_{K}^{0} \times \mathbb{R}^{K}$ is any infinite sequence of points in the graph $\Gamma_{\mathfrak{Z}}$ of $\mathfrak{Z}$ defined by (35) with the property that the price sequence $p^{\mathbb{N}}$ converges to a point $\bar{p}$ on the boundary of $P_{K}$ satisfying $\bar{p}^{J} \neq 0^{J}$-i.e. $\bar{p}_{j}>0$ for at least one $j \in J$. Then the sum $\sum_{g \in K} z_{g}^{n} \rightarrow+\infty$.

The feasibility condition 2 implies that the trader cannot offer to supply any commodity that has not been included in the effective announced endowment set $J$. The boundary condition 4 requires that, as one or more prices tend to zero, so total net demand over all commodities $g \in K$ tends to infinity whenever the "cheaper point" property holds-i.e. whenever at least one of commodities in the effective announced endowment subset $J$ has a positive price in the limit.

We remark that if the set $J$ is replaced by the larger set $\tilde{J} \supsetneq J$, then on the one hand the feasibility condition 2 is less restrictive in the sense that $z_{g}$ can be negative for $g \in \tilde{J} \backslash J$. On the other hand, the boundary condition is more restrictive in the sense that more components of any limit price vector $\bar{p}$ can be nonzero, implying that total net demand over all commodities $g \in K$ tends to infinity for more price sequences that converge to the boundary of the price simplex $P_{K}$. 
Note in particular that $J$-regularity does not require any revealed preference axiom or other rationality condition to be satisfied. Given any trading space $K \subseteq G$ and any endowment set $J \subseteq K$, let $\mathscr{Z}_{J}^{K}$ denote the set of all $J$-regular net demand correspondences $\mathfrak{Z}$. Then each $\mathfrak{Z} \in \mathscr{Z}_{J}^{K}$ can be identified uniquely by its graph $\Gamma_{\mathfrak{Z}}$, and so by continuity, with an appropriate subset of the family of all relatively closed subsets of $P_{K}^{0} \times \mathbb{R}^{K}$-or more precisely, of the set $\left\{(p, z) \in P_{K}^{0} \times \mathbb{R}^{K} \mid z \geqq-\left(\bar{e}^{J}, 0^{G \backslash J}\right)\right\}$.

Finally, given the probability measure $\gamma \in \mathscr{M}_{\tau}\left(L ; 2^{G}\right)$ and the trading space $K:=$ $G(\gamma)$ defined by (30), let

$$
\mathscr{Z}^{K}:=\bigcup_{J \subseteq K} \mathscr{Z}_{J}^{K}
$$

denote the domain of all net demand correspondences that are $J$-regular for some endowment set $J \subseteq K$.

\subsection{A statistical demand revelation mechanism}

We will now define a two-stage demand revelation mechanism.

1. At the first stage, each trader with label $\ell \in L$ reports an endowment set $H \subseteq G$. Moreover, following the discussion in Sect. 5.6, the traders' reports are assumed to determine an estimated distribution $\gamma \in \mathscr{M}_{\lambda}\left(L ; 2^{G}\right)$, and so a trading set $K=$ $G(\gamma) \subseteq G$ defined by (30), which is announced to each trader.

2. At the second stage, given the trading set $K=G(\gamma)$ defined by (30), each trader who has been recorded as having the pair $(\ell, H) \in L \times 2^{G}$ is next asked to report a $J$-regular net demand correspondence $\mathfrak{Z} \in \mathscr{Z}_{J}^{K}$, where $J=H \cap K \in 2^{K}$. Based on the list of all traders' recorded triples $(\ell, J, \mathfrak{Z})$, the market organizer is assumed to estimate the demand distribution $\zeta \in \mathscr{M}_{\gamma}\left(L \times 2^{K} ; \mathscr{Z}^{K}\right)$ which, by definition, satisfies the extra regularity condition

$$
\zeta\left(\left\{(\ell, J, \mathfrak{Z}) \in L \times 2^{K} \times \mathscr{Z}^{K} \mid \mathfrak{Z} \in \mathscr{Z}_{J}^{K}\right\}\right)=1
$$

In the following, for each distribution $\gamma \in \mathscr{M}_{\lambda}\left(L ; 2^{G}\right)$ with associated trading set $K=G(\gamma)$, let $\mathscr{M}_{\gamma}^{*}$ denote the subset of probability measures $\zeta$ over triples $(\ell, J, \mathfrak{Z})$ belonging to $\mathscr{M}_{\gamma}\left(L \times 2^{K} ; \mathscr{Z}^{K}\right)$ that satisfy (37).

Given the trading set $K \in 2^{G}$, player 0's task in the role of market organizer will be to devise a statistical demand revelation (or DR) mechanism that, for each estimated demand distribution $\zeta$ satisfying (37), selects: (a) a price vector $\bar{p}(\zeta) \in P_{K}^{0}$; and (b) a measurable allocation function

$$
L \times 2^{K} \times \mathscr{Z}^{K} \ni(\ell, J, \mathfrak{Z}) \mapsto \bar{z}^{\ell}(J, \mathfrak{Z} ; \zeta) \in \mathbb{R}^{K}
$$

that together constitute a market equilibrium satisfying, by definition:

- throughout the domain $L \times 2^{K} \times \mathscr{Z}^{K}$, the selection condition

$$
\bar{z}^{\ell}(J, \mathfrak{Z} ; \zeta) \in Z(\bar{p}(\zeta) ; \mathfrak{Z})
$$


- the market equilibrium condition

$$
0^{K}=\int_{L \times 2^{K} \times \mathscr{Z}^{K}} \bar{z}^{\ell}(J, \mathfrak{Z} ; \zeta) \zeta(\mathrm{d} \ell \times \mathrm{d} J \times \mathrm{d} \mathfrak{Z})
$$

A discussion of how our regularity conditions ensure existence of such a market equilibrium for each demand distribution $\zeta$ is deferred to Appendix 3.

\subsection{A statistical game of incomplete information}

The statistical DR mechanism specified in Sect. 6.3 generates a game of incomplete information in strategic form with:

1. the probability space $(L, \mathscr{L}, \lambda)$ of potential traders' labels;

2. the type space $T$ of potential traders' taste-endowment pairs $t=(\theta, e) \in \Theta \times E$;

3. a type distribution in the form of a probability measure $\tau \in \mathscr{M}_{\lambda}(L ; T)$ over labeltype pairs $(\ell, t) \in L \times T$ that describe potential traders;

4. for all traders, the common demand revelation strategy space

$$
\mathfrak{S}:=\bigcup_{H \in 2^{G}}\left(\{H\} \times \prod_{K \in 2^{G}} \mathscr{Z}_{H \cap K}^{K}\right)
$$

of pairs $\mathfrak{s}=\left(H, \mathfrak{Z}^{H}\right)$ which combine:

(a) an announced endowment set $H \subseteq G$;

(b) an entire profile

$$
\mathfrak{Z}^{H}=\left\langle\mathfrak{Z}_{K}\right\rangle_{K \in 2^{G}} \in \prod_{K \in 2^{G}} \mathscr{Z}_{H \cap K}^{K}
$$

specifying, for each possible trading set $K \subset G$ that the market organizer might announce, an associated $H \cap K$-regular net demand correspondence $\mathfrak{Z}_{K}$;

5. for each potential trader with label-type pair $(\ell, t) \in L \times T$, a pay-off function

$$
\mathfrak{S} \times \mathscr{M}_{\lambda}(L ; \mathfrak{S}) \ni(\mathfrak{s}, \sigma) \mapsto v^{\ell}(\mathfrak{s}, \sigma ; t)
$$

defined for every individual trader's demand revelation strategy $\mathfrak{s}=\left(H, \mathfrak{Z}^{H}\right) \in \mathfrak{S}$ and for every estimated measure $\sigma \in \mathscr{M}_{\lambda}(L ; \mathfrak{S})$ over traders' label-strategy pairs. Its value is given by

$$
v^{\ell}(\mathfrak{s}, \sigma ; t)=v^{\ell}\left(H, \mathfrak{Z}^{H}, \sigma ; t\right):=u\left(\bar{z}^{\ell}\left(H, \mathfrak{Z}_{K(\sigma)}^{H}, \zeta(\sigma)\right)+e ; \theta\right)
$$

where

(a) for the population of all potential traders, $K(\sigma):=G(\gamma(\sigma))$ denotes the trading set induced by the relevant marginal distribution $\gamma(\sigma):=\operatorname{marg}_{2^{G}} \sigma$ of the strategy measure $\sigma$; 
(b) given the demand set $H$ that the trader has already announced, $\mathfrak{Z}_{H \cap K(\sigma)}^{H}$ is the $H \cap K(\sigma)$-regular net demand correspondence that forms the appropriate component of the profile $\mathfrak{Z}^{H}$ defined by (42);

(c) for the population of all potential traders, $\zeta(\sigma) \in \mathscr{M}_{\gamma(\sigma)}^{*}$ denotes the induced estimated demand distribution $\zeta$ over the set $L \times 2^{G} \times \mathscr{Z}$ of all observable triples $(\ell, H, \mathfrak{Z})$ which, for every Borel set $V \subset L \times 2^{G} \times \mathscr{Z}^{K(\sigma)}$, satisfies

$$
\zeta(\sigma)(V):=\sigma\left(\left\{\left(\ell, H, \mathfrak{Z}^{H}\right) \in L \times \mathfrak{S} \mid\left(\ell, H, \mathfrak{Z}_{H \cap K(\sigma)}^{H}\right) \in V\right\}\right)
$$

\subsection{Walrasian demand revelation strategies}

Definition 2 A trader whose true type is $t=(\theta, e)$ has a non-empty true endowment set $H(e):=\left\{g \in G \mid e_{g}>0\right\}$, as well as for each trading space $K \subseteq G$ :

1. a true budget correspondence

$$
P_{K}^{0} \ni p \mapsto B_{K}(p ; e):=\left\{z \in \mathbb{R}^{K} \mid z \geqq-\left(e^{J}, 0^{K \backslash J}\right) \text { and } p z \leq 0\right\}
$$

where $J$ denotes the set $H(e) \cap K=\left\{g \in K \mid e_{g}>0\right\}$, whereas $0^{K \backslash J}$ denotes the zero vector in $\mathbb{R}^{K \backslash J}$, and $e^{J}=\left(e_{g}\right)_{g \in J} \in \mathbb{R}^{J}$.

2. a true Walrasian net demand correspondence $\hat{\mathfrak{Z}}_{K, t}: P_{K}^{0} \rightarrow \mathbb{R}^{K}$ defined by

$$
P_{K}^{0} \ni p \mapsto Z\left(p ; \hat{\mathfrak{Z}}_{K, t}\right):=\arg \max _{z}\left\{u(z+e ; \theta) \mid z \in B_{K}(p ; e)\right\}
$$

Let

$$
\hat{\mathfrak{s}}:=\left(H(e), \hat{\mathfrak{Z}}^{H(e)}\right)=\left(H(e),\left\langle\hat{\mathfrak{Z}}_{K, t}\right\rangle_{K \in 2^{G}}\right)
$$

denote this trader's (truthful) Walrasian demand revelation strategy.

By definitions (31) and (32), the domain $P_{K}^{0}$ excludes boundary points of the price simplex $P_{K}$. By the definition of $J$ as $\left\{g \in K \mid e_{g}>0\right\}$, one has $e^{J} \in \mathbb{R}_{++}^{J}$, so a standard argument based on the cheaper point lemma shows that this net demand correspondence is upper hemi-continuous.

In Appendix 2, it is shown that, provided that preferences are continuous and strictly monotone, the true Walrasian net demand correspondence $\hat{\mathfrak{Z}}_{K, t}$ also satisfies the boundary condition. Hence, $\hat{\mathfrak{J}}_{K, t}$ is $J$-regular according to the definition in Sect. 6.2.

\subsection{Strategyproofness}

For any actual trader whose true label-type pair is $(\ell, t) \in L \times T$, consider any change to the demand revelation strategy $\mathfrak{s}$ that this trader may choose from the strategy set $\mathfrak{S}$ defined by (41). Now, the measures $\gamma, \sigma$ and $\zeta(\sigma)$ defined in Sects. 6.1 and 6.4 all have a marginal distribution on $L$ equal to the non-atomic measure $\lambda$. It follows from the argument at the end of Appendix 1 that all these three measures are also non-atomic. So this change in one trader's demand revelation strategy $\mathfrak{s} \in \mathfrak{S}$ has no effect on: 
1. the estimated endowment distribution $\gamma \in \mathscr{M}_{\lambda}\left(L ; 2^{G}\right)$;

2. the associated trading space $K=G(\gamma) \subseteq G$ defined by (30);

3. the estimated demand distribution $\zeta(\sigma) \in \mathscr{M}_{\gamma}^{*}$ given by (45);

4. the associated Walrasian price vector $\bar{p}(\zeta) \in \mathbb{R}^{K}$ specified in Sect. 6.3.

Accordingly, given this announcement strategy $\left.\mathfrak{s}=\left(H,\left\langle\mathfrak{Z}_{H \cap K}^{H}\right)\right\rangle_{K \in 2^{G}}\right) \in \mathfrak{S}$ as well as the trading space $K=G(\gamma) \subseteq G$ and the associated endowment set $J=H \cap K$, whatever net demand vector $\bar{z}^{\ell}(J, \mathfrak{Z} ; \zeta) \in Z(\bar{p}(\zeta) ; \mathfrak{Z})$ is assigned to this trader by the demand selection specified in (39), it must belong to the value $Z\left(\bar{p}(\zeta) ; \mathfrak{Z}^{\ell, t}\right)$ of the announced $J$-regular net demand correspondence $\mathfrak{Z}^{\ell, t} \in \mathscr{Z}_{J}^{K}$ at the particular price $\bar{p}(\zeta)$, and so to the associated budget set $B^{K}(\bar{p}(\zeta))$ specified in (33).

Now, for this trader with true label-type pair $(\ell, t) \in L \times T$, any net demand vector $\bar{z}^{\ell}\left(\hat{\mathfrak{Z}}_{K, t}, \zeta\right)$ that is selected from the true Walrasian demand set $Z\left(p ; \hat{\mathfrak{J}}_{K, t}\right)$ must, by definition, be one of the most preferred among the net trade vectors in the budget set $B(\bar{p}(\zeta) ; e)$. In particular, it must be weakly preferred to the alternative net demand vector $\bar{z}^{\ell}\left(\mathfrak{Z}^{\ell, t}, \zeta\right)$. It follows that, no matter what the demand distribution $\zeta$ may be, this trader weakly prefers the truthful Walrasian demand revelation strategy $\hat{\mathfrak{s}} \in \mathfrak{S}$ to any allowed alternative $\mathfrak{s} \in \mathfrak{S}$. Moreover, when the alternative $\bar{z}^{\ell}\left(\mathfrak{Z}^{\ell, t}, \zeta\right)$ does not belong to $Z\left(p ; \hat{\mathfrak{Z}}_{K, t}\right)$, this preference is strict.

In this sense, truthfulness is always at least a weakly dominant strategy. So the demand revelation mechanism is indeed at least weakly strategyproof. Thus, in our limit economy with infinitely many traders, the market organizer can set up a demand revelation game in order to implement Walrasian equilibrium with a strategyproof mechanism.

Nevertheless, the objection raised in Sect. 3.4 still applies. Consider any trader with true label-type pair $(\ell, t) \in L \times T$, true endowment set $J$ and true Walrasian net demand correspondence $\hat{\mathfrak{Z}}_{K, t}$ as specified in Sect. 6.5. So far, no incentive has been provided for that trader to deliver the supply vector $y^{\ell}\left(J, \hat{\mathfrak{Z}}_{K, t}, \zeta\right)=-\left(\bar{z}^{\ell}\left(J, \hat{\mathfrak{Z}}_{K, t}, \zeta\right) \wedge 0\right)$ required to reach a Walrasian allocation.

\section{Demand revelation with warehousing}

\subsection{Traders' warehouse supplies}

The task now is to devise incentives for each potential trader with label-type pair $(\ell, t)=(\ell, \theta, e) \in L \times T$ not only to announce both the true endowment set $H(e) \in 2^{G}$ and true demand correspondence, but also to deliver the mandated supply vector to the warehouse system. To do so, we shall extend the DR mechanism of Sect. 6 into the kind of two-stage setting introduced in Sect. 4.1. Indeed, the estimated demand distribution $\zeta=\operatorname{marg}_{L \times Z} \sigma \in \mathscr{M}_{\gamma}^{*}$ given by (45) is used in order to determine, for each potential agent described by the recorded triple $(\ell, J, \mathfrak{Z}) \in L \times 2^{K} \times \mathscr{Z}^{K}$, where $J=H \cap K$, a mandated supply vector defined by

$$
\bar{y}^{\ell}(J, \mathfrak{Z} ; \zeta):=-\left(\bar{z}^{\ell}(J, \mathfrak{Z} ; \zeta) \wedge 0\right)
$$


This vector plays a key role in determining the outcome of the second-stage market mechanism. For each recorded triple $(\ell, J, \mathfrak{Z}) \in L \times 2^{K} \times \mathscr{Z}^{K}$, any corresponding actual agent is allowed to bring to the warehouse system any supply vector $y^{S} \in \mathbb{R}_{+}^{K}$ satisfying $y^{S} \leqq e$. This vector will not be accepted, however. Indeed, oversupplies are not allowed, so the accepted supply vector is $y:=y^{S} \wedge \bar{y}^{\ell}(J, \mathfrak{Z} ; \zeta)$. This exclusion makes it impossible for any trader to speculate that any good they oversupply could be bought back more cheaply in the second-stage market. ${ }^{5}$

These supply vectors that the actual traders choose to bring to the warehouse are assumed to determine a new strategic supply distribution in the form of an estimated joint probability measure

$$
\eta \in \mathscr{M}_{\zeta}\left(L \times 2^{K} \times \mathscr{Z}^{K} ; \mathbb{R}_{+}^{G}\right)
$$

over the space $L \times 2^{K} \times \mathscr{Z}^{K} \times \mathbb{R}_{+}^{G}$ of all recorded quadruples $(\ell, J, \mathfrak{Z}, y)$ of labels, endowment sets, demand correspondences, and warehouse supply vectors; this measure is assumed to be consistent with the appropriate first-stage demand distribution $\zeta$.

\subsection{Distinguishing deviant from compliant agents}

As in the corresponding mechanism with full information that was considered in Sect. 4, the conclusion of the mechanism with incomplete information distinguishes between:

compliant traders for whom $y=\bar{y}^{\ell}(J, \mathfrak{Z} ; \zeta)$;

deviant traders for whom $y<\bar{y}^{\ell}(J, \mathfrak{Z} ; \zeta)$.

Now, the normal compliant case occurs when, for the given joint distribution $\eta$ of demand correspondences and warehouse supply vectors, almost all traders are compliant-i.e.

$$
\eta\left(\left\{(\ell, J, \mathfrak{Z}, y) \in L \times 2^{K} \times \mathscr{Z}^{K} \times \mathbb{R}_{+}^{G} \mid y=\bar{y}^{\ell}(J, \mathfrak{Z} ; \zeta)\right\}\right)=1
$$

In this case:

1. any compliant trader is allowed to withdraw any commodity vector $x \in \mathbb{R}_{+}^{G}$ satisfying $x \leqq \bar{x}^{\ell}(J, \mathfrak{Z} ; \zeta):=\bar{z}^{\ell}(J, \mathfrak{Z} ; \zeta) \vee 0$;

2. any deviant trader is allowed to withdraw any commodity vector $x \in \mathbb{R}_{+}^{G}$ satisfying the budget constraint $p(\zeta) x \leq p(\zeta) y$, where $p(\zeta) y<p(\zeta) \bar{y}^{\ell}(J, \mathfrak{Z} ; \zeta)$ because $p(\zeta) \gg 0$ and $y<\bar{y}^{\ell}(J, \mathfrak{Z} ; \zeta)$.

The game ceases when all traders have completed their withdrawals, and any remaining stock in the warehouse has been thrown away.

\subsection{Warehouse supplies as endowments}

In the alternative deviant case when (51) is violated, there will be a non-negligible and observable discrepancy between:

\footnotetext{
5 My thanks to Michael Wickens for encouraging me to emphasize this point.
} 
- on the one hand, the measure $\eta$ generated by the actual warehouse deposits;

- on the other hand, the function $(\ell, J, \mathfrak{Z}) \mapsto \bar{y}^{\ell}(J, \mathfrak{Z} ; \zeta)$ specifying mandated warehouse deposits.

Indeed, let

$$
\mathbb{E}_{\eta}[y]:=\int_{L \times 2^{G} \times \mathscr{Z} \times \mathbb{R}_{+}^{G}} y \eta(\mathrm{d} \ell \times \mathrm{d} J \times \mathrm{d} \mathfrak{Z} \times \mathrm{d} y)
$$

denote the available mean warehouse supply vector per head, and let

$$
\mathbb{E}_{\zeta}\left[\bar{x}^{\ell}(J, \mathfrak{Z} ; \zeta)\right]=\mathbb{E}_{\zeta}\left[\bar{y}^{\ell}(J, \mathfrak{Z} ; \zeta)\right]:=\int_{L \times 2^{G} \times \mathscr{Z}} \bar{y}^{\ell}(J, \mathfrak{Z} ; \zeta) \zeta(\mathrm{d} \ell \times \mathrm{d} J \times \mathrm{d} \mathfrak{Z})
$$

denote the mean warehouse demand vector per head. Because $y<\bar{y}^{\ell}(J, \mathfrak{Z} ; \zeta)$ on a non-negligible set, one has $\mathbb{E}_{\eta}[y]<\mathbb{E}_{\zeta}\left[\bar{x}^{\ell}(J, \mathfrak{Z} ; \zeta)\right]$-i.e. when aggregated over all traders, whether actual or potential, the mean supply is almost surely insufficient to meet the mean demand.

In order to deal with this discrepancy and to deter the failures to deliver the mandated warehouse deposits that must have occurred, the market organizer will institute a new second-stage demand revelation mechanism. At the beginning of this second stage, each potential trader is described by the combination $(\ell, t, J, \mathfrak{Z}, y)$ of a label $\ell \in L$, a type $t=(\theta, e) \in T$, a recorded endowment set $J \in 2^{K}$, a recorded net demand correspondence $\mathfrak{Z} \in \mathscr{Z}_{J}^{K}$, and the trader's chosen warehouse supply vector $y \in \mathbb{R}_{+}^{G}$. The endowment vector $e$ is no longer relevant, however, because no trader is allowed to deposit any more of any good into the warehouse system. Instead, the predetermined supply vector $y$ is treated as an endowment, even though it has already been delivered to the warehouse system. Each trader, however, can choose how much of the effective endowment $y$ to reclaim for personal consumption, depending on the second-period price vector.

\subsection{A second-stage commodity space}

The second-stage demand revelation mechanism will work with the restricted trading space

$$
G^{+}(\eta):=\left\{g \in G \mid \mathbb{E}_{\eta}\left[y_{g}\right]>0\right\}
$$

consisting of commodities for which the mean warehouse deposit per trader is positive. At the second stage, trade will take place only in these commodities; traders' warehouse deposits $y_{g}$ of all other goods $g \in K \backslash G^{+}(\eta)$ are returned in their entirety to their depositors, in order to ensure that no trader ever loses from making those deposits.

From now on, therefore, we consider the commodity space $\mathbb{R}^{G^{+}(\eta)}$, as well as the new version

$$
Q:=\left\{q \in \mathbb{R}_{+}^{G^{+}(\eta)} \mid \sum_{g \in G} q_{g}=1\right\}
$$


of the unit simplex $P \subset \mathbb{R}^{G}$ of semi-positive normalized price vectors defined by (1) of Sect. 2.1, together with the new version

$$
Q^{0}:=\left\{q \in \mathbb{R}_{++}^{G^{+}(\eta)} \mid \sum_{g \in G} q_{g}=1\right\}
$$

of the relative interior $P^{0} \subset P$ defined by (2).

For each supply vector $y=\left\langle y_{g}\right\rangle_{g \in K} \in \mathbb{R}_{+}^{K}$ that a trader might supply, we introduce the notation

$$
y^{+}:=\left\langle y_{g}\right\rangle_{g \in G^{+}(\eta)} \in \mathbb{R}_{+}^{G^{+}(\eta)}
$$

for the subvector whose components correspond to goods that the trader supplies in positive amounts.

\subsection{Second-stage regular demands}

As the second stage starts, each actual trader has a record in the form of a quadruple $\left(\ell, J, \mathfrak{Z}, y^{+}\right)$that combines an observable label $\ell \in L$, a reported endowment set $H \in 2^{G}$ such that $J=H \cap K$, a reported $J$-regular net demand correspondence $\mathfrak{Z} \in \mathscr{Z}_{J}^{K}$, and a chosen warehouse supply vector $y^{+} \in \mathbb{R}_{+}^{G^{+}(\eta)}$ defined by (57) for those commodities $g \in G^{+}(\eta)$ that will be traded at the second stage. For each price vector $q \in Q^{0}$, the trader's associated budget set of permissible demand vectors $x^{+}$ in the trading space $\mathbb{R}^{G^{+}(\eta)}$ is taken to be

$$
B_{X}\left(q ; y^{+}, \eta\right):=\left\{x^{+} \in \mathbb{R}_{+}^{G^{+}(\eta)} \mid q x^{+} \leq q y^{+}\right\}
$$

For each price vector $q \in Q^{0}$, this limits the trader's allowable expenditure $q x^{+}$on warehouse deliveries $x^{+} \in \mathbb{R}_{+}^{G^{+}(\eta)}$ to the value $q y^{+}$of the trader's own recorded warehouse supply vector.

Let $z^{+}:=x^{+}-y^{+} \in \mathbb{R}^{G^{+}(\eta)}$ denote the trader's second-stage net trade vector. For each price vector $q \in Q^{0}$, the budget set (58) can then be expressed in the equivalent form

$$
B^{+}\left(q ; y^{+}, \eta\right):=\left\{z^{+} \in \mathbb{R}^{G^{+}(\eta)} \mid z^{+} \geqq-y^{+} \text {and } q z^{+} \leq 0\right\}
$$

This is obviously analogous to the endowment constrained budget set defined by (4) in Sect. 3.1.

Given the supply vector $y^{+} \in \mathbb{R}_{+}^{G^{+}(\eta)}$, let

$$
J\left(y^{+}\right):=\left\{g \in G^{+}(\eta) \mid y_{g}^{+}>0\right\}
$$

denote the set of goods that are supplied in positive amounts. Then let $\mathscr{Z}_{y^{+}}^{+}$denote the set of all $J\left(y^{+}\right)$-regular net demand correspondences $\mathfrak{Z}^{+}$. These are multifunctions

$$
Q^{0} \ni q \mapsto Z^{+}\left(q ; \mathfrak{Z}^{+}\right) \subseteq B^{+}\left(q ; y^{+}, \eta\right) \subset \mathbb{R}^{G^{+}(\eta)}
$$


which satisfy the budget exhaustion, feasibility, continuity, and boundary conditions set out in Sect. 6.2, but with the commodity set $K$ replaced by $G^{+}(\eta)$, and with the endowment set $J$ replaced by $J\left(y^{+}\right)$.

Given the vector $\bar{e}$ of upper bounds specified in (25), as well as the supply distribution $\eta$, let $\bar{e}^{+}:=\left(\bar{e}_{g}\right)_{g \in G^{+}(\eta)} \in \mathbb{R}^{G^{+}(\eta)}$ denote the vector of uniform upper bounds on traders' endowments, restricted to the set $G^{+}(\eta)$ of goods to be traded at the second stage. Because each trader's warehouse deposit vector $y$ must satisfy the feasibility constraint $y \leqq e \leqq \bar{e}$, it follows that the restriction $y^{+}=\left(y_{g}\right)_{g \in G^{+}(\eta)} \in \mathbb{R}^{G^{+}(\eta)}$ must satisfy $y^{+} \leqq \bar{e}^{+}$. Accordingly, let

$$
\mathscr{Z}^{+}:=\cup_{0 \leqq y^{+}} \leqq \bar{e}^{+} \mathscr{Z}_{y^{+}}^{+}
$$

denote the domain of all regular net demand correspondences $\mathfrak{Z}^{+}$-i.e. all net demand correspondences $\mathfrak{Z}^{+}$that are $y^{+}$-regular for some feasible $y^{+} \leqq \bar{e}^{+}$.

\subsection{A second-stage demand revelation mechanism}

As the second stage starts, player 0 has a record for each actual trader taking the form of a quadruple $(\ell, J, \mathfrak{Z}, y)$ that combines an observable label $\ell \in L$, a reported endowment set $J \in 2^{K}$, a reported $J$-regular net demand correspondence $\mathfrak{Z} \in \mathscr{Z}_{J}^{K}$, and a supply vector $y \in \mathbb{R}_{+}^{G}$. Furthermore, as in (50), player 0 has already used all these records in order to estimate the supply distribution $\eta$ over these quadruples.

We now specify a second-stage DR mechanism along the lines of the first-stage DR mechanism set out in Sect. 7.1. Specifically:

1. First, player 0 as statistician uses definitions (54) and (57) to compute the set $G^{+}(\eta)$ of commodities to be traded, as well as the restriction $y^{+} \in \mathbb{R}_{+}^{G^{+}(\eta)}$ of each trader's chosen warehouse supply vector $y \in \mathbb{R}^{K}$ to the subset $G^{+}(\eta)$ of commodities $g \in G$.

2. Each trader with recorded quadruple $\left(\ell, J, \mathfrak{Z}, y^{+}\right)$is required to report to player 0 a $J\left(y^{+}\right)$-regular second-stage net demand correspondence $Q^{0} \ni q \mapsto Z^{+}(q) \in$ $\mathbb{R}^{G^{+}(\eta)}$, or $\mathfrak{Z}^{+} \in \mathscr{Z}^{+}$.

3. To each trader's previous record $(\ell, J, \mathfrak{Z}, y) \in L \times 2^{K} \times \mathscr{Z}^{K} \times \mathbb{R}_{+}^{G}$, player 0 appends the reported second-stage net demand correspondence $\mathfrak{Z}^{+} \in \mathscr{Z}^{+}$, thus forming the quintuple $\left(\ell, J, \mathfrak{Z}, y, \mathfrak{Z}^{+}\right)$.

4. Based on all the quintuples recorded at the previous step, player 0 estimates the joint distribution $\zeta^{+} \in \mathscr{M}_{\eta}\left(L \times 2^{K} \times \mathscr{Z}^{K} \times \mathbb{R}_{+}^{G} ; \mathscr{Z}^{+}\right)$. Regularity of each trader's announced net demand correspondence implies that $\zeta^{+}$must satisfy the additional restriction

$$
\zeta^{+}\left(\left\{\left(\ell, J, \mathfrak{Z}, y, \mathfrak{Z}^{+}\right) \in L \times 2^{K} \times \mathscr{Z} \times \mathbb{R}_{+}^{G} \times \mathscr{Z}^{+} \mid \mathfrak{Z}^{+} \in \mathscr{Z}_{y^{+}}^{+}\right\}\right)=1
$$

5. Depending on the distribution $\zeta^{+} \in \mathscr{M}_{\eta}\left(L \times 2^{K} \times \mathscr{Z}^{K} \times \mathbb{R}_{+}^{G} ; \mathscr{Z}^{+}\right)$that was estimated at Step 4, player 0 determines both a second-stage price vector $\bar{q}\left(\zeta^{+}\right)$ and a second-stage allocation in the form of an integrable function 
$L \times 2^{K} \times \mathscr{Z}^{K} \times \mathbb{R}_{+}^{G} \ni\left(\ell, J, \mathfrak{Z}, y, \mathfrak{Z}^{+}\right) \mapsto \tilde{z}^{\ell}\left(J, \mathfrak{Z}, y, \mathfrak{Z}^{+} ; \zeta^{+}\right) \in \mathbb{R}^{G^{+}(\eta)}$

that together satisfy:

(a) the selection condition

$$
\begin{aligned}
& \tilde{z}^{\ell}\left(J, \mathfrak{Z}, y, \mathfrak{Z}^{+} ; \zeta^{+}\right) \in Z^{+}\left(q\left(\zeta^{+}\right) ; \mathfrak{Z}^{+}\right) \subseteq \mathbb{R}^{G^{+}(\eta)} \\
& \quad \text { for } \zeta^{+}-\text {a.e. }\left(\ell, J, \mathfrak{Z}, y, \mathfrak{Z}^{+}\right) \in L \times 2^{K} \times \mathscr{Z} \times \mathbb{R}_{+}^{G} \times \mathscr{Z}^{+}
\end{aligned}
$$

(b) the market clearing condition

$$
0=\int_{L \times 2^{K} \times \mathscr{Z} \times \mathbb{R}_{+}^{G} \times \mathscr{Z}+} \tilde{z}^{\ell}\left(J, \mathfrak{Z}, y, \mathfrak{Z}^{+} ; \zeta^{+}\right) \zeta^{+}\left(\mathrm{d} \ell \times \mathrm{d} J \times \mathrm{d} Z \times \mathrm{d} y \times \mathrm{d} \mathfrak{Z}^{+}\right)
$$

6. Each trader with the recorded quintuple $\left(\ell, J, \mathfrak{Z}, y, \mathfrak{Z}^{+}\right)$is allowed to claim from the warehouse any commodity vector $x^{+} \in \mathbb{R}_{+}^{G^{+}(\eta)}$ satisfying $x^{+} \leqq$ $\tilde{x}^{\ell}\left(J, \mathfrak{Z}, y, \mathfrak{Z}^{+} ; \zeta^{+}\right)$, where

$$
\tilde{x}^{\ell}\left(J, \mathfrak{Z}, y, \mathfrak{Z}^{+} ; \zeta^{+}\right):=\tilde{z}^{\ell}\left(J, \mathfrak{Z}, y, \mathfrak{Z}^{+} ; \zeta^{+}\right)+y^{+}
$$

denotes the demand allowance corresponding to the assigned net trade vector; the game ceases when all traders have made their withdrawals within their specified limit, after which player 0 disposes of any remaining surplus.

For each $\zeta^{+}$in the relevant domain $\mathscr{M}_{\eta}\left(L \times 2^{K} \times \mathscr{Z} \times \mathbb{R}_{+}^{G} ; \mathscr{Z}^{+}\right)$of second-stage demand distributions, the existence theorem of Appendix 3 can be applied to show that there is a price vector $\tilde{q}\left(\zeta^{+}\right) \in Q^{0}$ and an allocation function which together satisfy both (65) and (66).

\section{A mechanism in twenty-two steps}

\subsection{Defining the extensive form game}

The discussion so far has been sketched an extensive form game ${ }^{6}$ whose players are:

- the countably infinite set of traders $k \in \mathbb{N}$ whose respective label-type pairs $\left(\ell^{k}, t^{k}\right) \in L \times T$ are independently drawn from the probability measure $\tau \in$ $\mathscr{M}_{\lambda}(L ; T)$;

- player 0 , who manages the market by combining all five roles of recording clerk, statistician, auctioneer, principal, and warehouse manager.

This game can be set out in full detail using the following twenty-two steps:

1. Player 0 announces a finite set $G$ of all possible goods that are candidates for trade.

\footnotetext{
${ }^{6}$ Strictly speaking, according to the usual definition of an extensive form game, players are allowed to move only one at a time. Our formulation follows Dubey and Kaneko (1984) and others in allowing many players to move simultaneously.
} 
2. Successive traders numbered $k=1,2, \ldots$ appear in turn before player 0 , and signal their intention to participate in the market by reporting a non-empty endowment set $H^{k} \subseteq G$, whereupon each is assigned a random label $\ell^{k}$ drawn independently from the constant probability measure $\lambda$ on $L$.

3. Player 0 as clerk records the infinite list of all reported pairs $\left(\ell^{k}, H^{k}\right) \in L \times 2^{G}$.

4. Player 0 as statistician estimates the joint endowment distribution $\gamma \in \mathscr{M}_{\lambda}\left(L ; 2^{G}\right)$ of all the pairs $(\ell, H) \in L \times 2^{G}$ that were recorded at Step 3 .

5. Given the endowment distribution $\gamma$ that was estimated at Step 4, player 0, now acting as market manager:

(a) constructs for each good $g \in G$ the set

$$
L_{g}:=\left\{(\ell, H) \in L \times 2^{G} \mid g \in H\right\}
$$

of pairs where the endowment set $H$ includes good $g$;

(b) calculates and then publicly announces the trading set

$$
K:=G(\gamma):=\left\{g \in G \mid \gamma\left(L_{g}\right)>0\right\}
$$

6. Each trader with record $(\ell, H) \in L \times 2^{G}$, who therefore faces the relevant trading set $J:=H \cap K$, is required by player 0 to report a first-stage $J$-regular net demand correspondence $\mathfrak{Z} \in \mathscr{Z}_{J}^{K}$. Any trader with record $(\ell, H)$ who refuses to report such a correspondence is penalized by being recorded as having the autarkic net demand correspondence $\mathfrak{Z}^{\text {aut }}$ that satisfies $Z\left(p, \mathfrak{Z}^{\text {aut }}\right)=\left\{0^{K}\right\}$ for all $p \in P_{K}^{0}$.

7. Player 0, acting as clerk, updates each trader's pair $(\ell, H) \in L \times 2^{G}$ that was recorded at Step 3 by:

- first replacing $H$ with $J:=H \cap K$;

- then appending to $(\ell, H)$ this trader's reported net demand correspondence $\mathfrak{Z} \in \mathscr{Z}_{J}^{K}$.

The resulting new record is a triple $(\ell, J, \mathfrak{Z}) \in L \times 2^{K} \times \mathscr{Z}^{K}$.

8. Player 0 , acting as statistician, estimates the joint distribution $\zeta \in \mathscr{M}_{\gamma}^{*}$ of all the triples $(\ell, J, \mathfrak{Z}) \in L \times 2^{K} \times \mathscr{Z}^{K}$ recorded at Step 7. Because of Step 6, this distribution must satisfy the regularity condition (37).

9. Conditional on the demand distribution $\zeta$ that was estimated at Step 8, player 0, acting as market equilibrator, determines:

(a) a first-stage price vector $\bar{p}(\zeta) \in P_{K}^{0}$;

(b) an allocation in the form of an integrable selection

$$
L \times 2^{K} \times \mathscr{Z}^{K} \ni(\ell, J, \mathfrak{Z}) \mapsto \bar{z}^{\ell}(J, \mathfrak{Z} ; \zeta) \in Z(\bar{p}(\zeta) ; \mathfrak{Z}) \subset \mathbb{R}^{K}
$$

from the $J$-regular demand correspondences announced at Step 6. These two must satisfy the market clearing condition (40).

10. Player 0, acting as warehouse manager, opens up the warehouse to accept traders' deposits, and then, as each trader arrives at the warehouse, player 0 , acting as clerk:

(a) consults each trader's triple $(\ell, J, \mathfrak{Z}) \in L \times 2^{K} \times \mathscr{Z}^{K}$ that was recorded at Step 6; 
(b) uses the allocation found at Step 9(b) in order to calculate and then announce to each trader the mandated warehouse supply vector

$$
\bar{y}^{\ell}(J, \mathfrak{Z} ; \zeta):=-\left[\bar{z}^{\ell}(J, \mathfrak{Z} ; \zeta) \wedge 0\right] \in \mathbb{R}_{+}^{K}
$$

11. Each trader who has the unobserved endowment vector $e$ brings to the warehouse any supply vector $y^{S} \in \mathbb{R}_{+}^{K}$ satisfying $y^{S} \leqq e$.

12. Player 0 , acting as warehouse manager, when confronted by a trader who is described by the triple $(\ell, J, \mathfrak{Z})$ recorded at Step 7, and who at Step 11 brings the supply $y^{S}$ to the warehouse, accepts as deposit the vector $y:=y^{S} \wedge \bar{y}^{\ell}(J, \mathfrak{Z} ; \zeta)$.

13. Player 0, acting as clerk, appends the deposit vector $y$ accepted at Step 12 to the triple $(\ell, J, \mathfrak{Z})$ recorded at Step 7, so the trader's updated record takes the form of a quadruple

$$
(\ell, J, \mathfrak{Z}, y) \in L \times 2^{K} \times \mathscr{Z}^{K} \times \mathbb{R}_{+}^{G}
$$

14. Player 0 , acting as statistician, uses the list of all the quadruples $(\ell, J, \mathfrak{Z}, y)$ that were recorded at step 13 in order to estimate:

(a) the corresponding supply distribution $\eta \in \mathscr{M}_{\zeta}\left(L \times 2^{K} \times \mathscr{Z}^{K} ; \mathbb{R}_{+}^{G}\right)$;

(b) the measure $\eta(C(\zeta))$ of the set

$$
C(\zeta):=\left\{(\ell, J, \mathfrak{Z}, y) \in L \times 2^{K} \times \mathscr{Z}^{K} \times \mathbb{R}_{+}^{G} \mid y=\bar{y}^{\ell}(J, \mathfrak{Z} ; \zeta)\right\}
$$

of all compliant quadruples $(\ell, J, \mathfrak{Z}, y)$.

15. Consider first the compliant case when $\eta(C(\zeta))=1$. Here, player 0 acting as warehouse manager allows each trader with the quadruple $(\ell, J, \mathfrak{Z}, y)$ that was recorded at Step 13 to withdraw any demand vector $x \geqq 0$ satisfying:

(a) in case $(\ell, J, \mathfrak{Z}, y)$ is a compliant quadruple in $C$, the rationing constraint $x \leqq \bar{x}^{\ell}(J, \mathfrak{Z} ; \zeta):=\left[\bar{z}^{\ell}(J, \mathfrak{Z} ; \zeta) \vee 0\right] \in \mathbb{R}_{+}^{G}$, where $\bar{z}^{\ell}(J, \mathfrak{Z} ; \zeta)$ is specified at Step 9 ;

(b) in case $(\ell, J, \mathfrak{Z}, y)$ is a deviant quadruple outside $C$, the budget constraint $\bar{p}(\zeta) x \leq \bar{p}(\zeta) y$, where $\bar{p}(\zeta)$ is the market clearing price in $P^{0}$ that was chosen at Step 8.

In this case where almost all traders are compliant, player 0 follows this Step 15 by jumping directly to the concluding Step 22 of the game.

16. Alternatively, in the deviant case when $\eta(C)<1$, player 0 constructs the set $G^{+}(\eta)$ defined by (54) of goods $g \in K$ whose mean supply per trader is positive, thus allowing good $g$ to be traded in the second-stage market. If the number of such goods satisfies \# $G^{+}(\eta)<2$, then trade is impossible. Therefore, in this case player 0 returns each deposit of every good to its depositor and declares that there is autarky.

Otherwise, if $\# G^{+}(\eta) \geq 2$, then player 0 :

- announces the set $G^{+}(\eta)$;

- returns any deposits of goods $g \in G \backslash G^{+}(\eta)$ to the depositors;

- starting at Step 17, institutes a second-stage demand revelation mechanism like that specified in Sect. 7.6. 
17. Each trader, given the already recorded deposit vector $y^{+} \in \mathbb{R}_{+}^{G^{+}(\eta)}$, is required to report to player 0 a $J\left(y^{+}\right)$-regular second-stage net demand correspondence $Q^{0} \ni q \mapsto Z^{+}(q) \in \mathbb{R}^{G^{+}(\eta)}$, or $\mathfrak{Z}^{+} \in \mathscr{Z}^{+}$.

Any trader with recorded quadruple $\left(\ell, J, \mathfrak{Z}, y^{+}\right)$who fails to report such a net demand correspondence is recorded as having the autarkic net demand correspondence $\left(\mathfrak{Z}^{+}\right)^{\text {aut }}$ that satisfies $Z^{+}\left(q,\left(\mathfrak{Z}^{+}\right)^{\text {aut }}\right)=\left\{0^{G^{+}(\eta)}\right\}$ for all $q \in Q^{0}$.

18. Player 0 , acting as clerk, updates each trader's quadruple $(\ell, J, \mathfrak{Z}, y) \in L \times 2^{K} \times$ $\mathscr{Z}^{K} \times \mathbb{R}_{+}^{G}$ that was recorded at Step 13 by appending the same trader's secondstage net demand correspondence $\mathfrak{Z}^{+} \in \mathscr{Z}^{+}$that was recorded at Step 17, thus extending the trader's record to the quintuple $\left(\ell, J, \mathfrak{Z}, y, \mathfrak{Z}^{+}\right) \in L \times 2^{K} \times \mathscr{Z}^{K} \times$ $\mathbb{R}^{G} \times \mathscr{Z}^{+}$.

19. Given all the quintuples $\left(\ell, J, \mathfrak{Z}, y, \mathfrak{Z}^{+}\right) \in L \times 2^{K} \times \mathscr{Z}^{K} \times \mathbb{R}_{+}^{G} \times \mathscr{Z}^{+}$that were recorded at Step 18, player 0 acting as statistician estimates the associated demand distribution $\zeta^{+} \in \mathscr{M}_{\eta}\left(L \times 2^{K} \times \mathscr{Z}^{K} \times \mathbb{R}_{+}^{G} ; \mathscr{Z}^{+}\right)$.

20. Given the distribution $\zeta^{+}$estimated at step 19, player 0 , acting as market equilibrator, next determines:

(a) a second-stage price vector $\tilde{q}\left(\zeta^{+}\right) \in Q^{0}$;

(b) from the $J\left(y^{+}\right)$-regular demand correspondences announced at Step 18, a measurable selection specified by (65), which also satisfies the market clearing condition (66).

21. Each trader with the quintuple $\left(\ell, J, \mathfrak{Z}, y, \mathfrak{Z}^{+}\right)$that was recorded at Step 18 , and so with the second-stage net trade vector $\tilde{z}^{\ell}\left(\mathfrak{Z}, \zeta, y, \mathfrak{Z}^{+} ; \zeta^{+}\right)$that was specified in Step 20, is allowed to withdraw from the warehouse any demand vector $x^{+} \geqq 0$ which satisfies $x^{+} \leqq \tilde{x}^{\ell}\left(J, \mathfrak{Z}, y, \mathfrak{Z}^{+} ; \zeta^{+}\right)$for the allowance specified by (67).

22. When all traders have made their allowed withdrawals, player 0 disposes of any remaining surplus.

\subsection{Two rounds of eliminating dominated strategies}

Following the discussion in Sect. 4.9 for the case of an economy with complete information and a finite number of traders, suppose that player 0 , as a market manager who combines the five roles of recording clerk, statistician, auctioneer, principal, and warehouse manager, is committed to a specified sequence of actions at Steps 1, 3-5, $7-10,12-16,18-20$, and 22 of the extensive form game set out in Sect. 8.1. Conditional upon these commitments, there is a game of incomplete information with a countably infinite set of traders who must all select a five-part strategy. The first three parts specify the trader's moves at Steps 2, 6, and 11. Furthermore, in case the spot market needs to reopen after a non-negligible set of traders have failed to deliver at Step 11 the supply vectors which player 0 had mandated at Step 10, there are two extra parts that specify the trader's moves at Steps 17 and 21 of the extensive form continuation game that arises in this "non-compliant" case.

This extensive form game will be analysed using backward recursion, starting at the penultimate Step 21 . There, because preferences are assumed to be strictly monotone, the obvious strictly dominant strategy for each trader is to withdraw the full allowance $\tilde{x}^{\ell}\left(J, \zeta^{+}, \mathfrak{Z}, \zeta, y, \mathfrak{Z}^{+}\right)$defined by (67). 
Next, working backwards to the demand revelation mechanism at Step 17, we recognize that by then each trader's endowment has effectively become the vector $y^{+} \in$ $\mathbb{R}_{+}^{G^{+}(\eta)}$ which has already been deposited in the warehouse system. Then the argument in Sect. 6.6 shows that revealing one's true Walrasian demand correspondence is a weakly dominant strategy.

\subsection{The warehouse supply subgame: strategies}

Moving further backwards through the extensive form game between all the traders, we come next to the key Step 11, which is reached after:

1. all the traders have already announced both endowment sets $H$ at Step 2 and net demand correspondences $\mathfrak{Z}$ at Step 6;

2. these announcements have been recorded as Step 7 and then used at Step 8 in order to estimate the demand measure $\zeta \in \mathscr{M}_{\gamma}^{*}$;

3. each trader with the recorded triple $(\ell, J, \mathfrak{Z}) \in L \times 2^{K} \times \mathscr{Z}^{K}$, where $J=H \cap K$, has been informed at Step 10 of both the mandated demand vector $\bar{x}^{\ell}(J, \mathfrak{Z} ; \zeta)$ and the mandated supply vector $\bar{y}^{\ell}(J, \mathfrak{Z} ; \zeta)$.

At Step 11, as already explained in Sect. 8.2, using backward recursion to eliminate dominated strategies leaves each trader with only the strategy of truthfully revealing their Walrasian net demand correspondence at Step 17, and then of withdrawing all they are allowed at Step 21. So, given the history $\zeta$ that player 0 estimates at Step 8, as well as the key variables that player 0 specifies at Steps 9 and 10, the traders at Step 11 are confronted with a one-shot continuation game of incomplete information, denoted by $\mathfrak{G}(\zeta)$, for which we now determine the strategy sets and pay-off functions.

In this game $\mathfrak{G}(\zeta)$, each trader's full description involves the recorded triple $(\ell, J, \mathfrak{Z}) \in L \times 2^{K} \times \mathscr{Z}^{K}$ supplemented by the hidden type $t=(\theta, e)$. Though the type remains hidden, the endowment vector $e$ limits what warehouse supply vector $y \in \mathbb{R}_{+}^{K}$ the trader can choose, whereas the mandated supply vector $\bar{y}^{\ell}(J, \mathfrak{Z} ; \zeta)$ limits what the warehouse manager will accept. Accordingly, we introduce the notation

$$
\bar{y}_{e}^{\ell}(J, \mathfrak{Z}, \zeta):=e \wedge \bar{y}^{\ell}(J, \mathfrak{Z} ; \zeta)
$$

for the upper bound on the vector of supplies that the trader can deliver to the warehouse system, and then

$$
Y_{e}^{\ell}(J, \mathfrak{Z}, \zeta):=\left\{y \in \mathbb{R}_{+}^{K} \mid y \leqq \bar{y}_{e}^{\ell}(J, \mathfrak{Z}, \zeta)\right\}
$$

for the strategy set of a trader described by the quadruple $(\ell, J, t, \mathfrak{Z})$ with hidden type $t=(\theta, e)$, when the estimated demand measure is $\zeta$. Though it depends on the hidden endowment $e$, the constraint $y \leqq e$ is obviously self-enforcing when applied to actual rather than to promised deliveries.

\subsection{The warehouse supply subgame: pay-offs}

In the game $\mathfrak{G}(\zeta)$ with strategy sets specified by (72), the traders' choices at Step 11 of the extensive form game are assumed to determine the supply distribution $\eta \in$ 
$\mathscr{M}_{\zeta}\left(L \times 2^{K} \times \mathscr{Z}^{K} ; \mathbb{R}_{+}^{G}\right)$ that player 0 estimates at Step 14 . The estimated supply distribution $\eta$ determines player 0's next choices at Steps 15 and 16. Hence, each trader's pay-off function in the subgame $\mathfrak{G}(\zeta)$ depends not only on the trader's own supply vector $y$, but also on the measure $\eta$, as well as on the trader's own hidden type $t=(\theta, e)$ and the previously recorded triple $(\ell, J, \mathfrak{Z})$. Of course, since the measure $\eta$ is non-atomic, no trader's choice of supply vector $y$ can affect it. This leaves two cases to consider, depending on whether the proportion of compliant agents satisfies $\eta(C)=1$ or $\eta(C)<1$.

In the compliant case when $\eta(C)=1$, player 0 is committed to follow Step 15 . The typical trader described by the quadruple $(\ell, J, t, \mathfrak{Z})$ has a pay-off that depends then on whether:

- $y=\bar{y}^{\ell}(J, \mathfrak{Z} ; \zeta)$. This is only possible when $\bar{y}^{\ell}(J, \mathfrak{Z} ; \zeta) \leqq e$. Then the trader is compliant and receives the pay-off

$$
w^{\ell}\left(\bar{y}^{\ell}(J, \mathfrak{Z} ; \zeta), \zeta ; \theta, e, \mathfrak{Z}\right)=u\left(\bar{z}^{\ell}(J, \mathfrak{Z} ; \zeta)+e ; \theta\right)
$$

from the mandated net demand vector $\bar{z}^{\ell}(J, \mathfrak{Z} ; \zeta)$.

$-y<\bar{y}^{\ell}(J, \mathfrak{Z} ; \zeta)$. Then the trader is deviant and receives the pay-off

$$
w^{\ell}(y, \zeta ; \theta, e, \mathfrak{Z})=\max _{z}\left\{u(z+e ; \theta) \mid z \geqq-\bar{y}^{\ell}(J, \mathfrak{Z} ; \zeta) \text { and } p(\zeta) z \leq 0\right\}
$$

But the mandated net demand vector $\bar{z}^{\ell}(J, \mathfrak{Z} ; \zeta)$ solves the maximization problem in (74) when the constraint $z \geqq-\bar{y}^{\ell}(J, \mathfrak{Z} ; \zeta)$ is replaced by the constraint $z \geqq-e$. When the trader is compliant and so $\bar{y}^{\ell}(J, \mathfrak{Z} ; \zeta) \leqq e$, the constraint $z \geqq-e$ is weaker. Hence

$$
w^{\ell}\left(\bar{y}^{\ell}(J, \mathfrak{Z} ; \zeta), \zeta ; \theta, e, \mathfrak{Z}\right) \geq w^{\ell}(y, \zeta ; \theta, e, \mathfrak{Z})
$$

for all $y<\bar{y}^{\ell}(J, \mathfrak{Z} ; \zeta)$. Since this is true for all measures $\zeta$ and all compliant $\eta$, it implies that compliance is a weakly dominant strategy in this case.

In the alternative case when $\eta(C(\zeta))<1$, player 0 is committed to follow Step 16, and to reopen markets for goods $g \in G^{+}(\eta)$. We consider this case next.

\subsection{Dominant warehouse supply strategies}

Consider any joint distribution $\zeta^{+} \in \mathscr{M}_{\eta}\left(L \times 2^{K} \times \mathscr{Z}^{K} \times \mathbb{R}_{+}^{G} ; \mathscr{Z}^{+}\right)$with associated price vector $\tilde{q}\left(\zeta^{+}\right)$that player 0 is committed to choose at Step 20. Each trader's anticipated pay-off in the game $\mathfrak{G}(\zeta)$ will then depend not only on the trader's own supply vector $y$ and the measure $\zeta^{+}$, but also on the trader's own previously recorded triple $(\ell, J, \mathfrak{Z})$, as well as the hidden type $t=(\theta, e)$. Hence, this trader's pay-off can be written as

$$
w^{\ell}\left(y, \zeta^{+} ; \theta, e, J, \mathfrak{Z}\right)=\max _{x}\left\{u(x-y+e ; \theta) \mid x \geqq 0 \text { and } \tilde{q}\left(\zeta^{+}\right) x \leq \tilde{q}\left(\zeta^{+}\right) y\right\}
$$

Introducing the change of variable $z=x-y$, with $y \leqq e$ fixed, one can rewrite (75) as 


$$
w^{\ell}\left(y, \zeta^{+} ; \theta, e, J, \mathfrak{Z}\right)=\max _{z}\left\{u(z+e ; \theta) \mid z \geqq-y \text { and } \tilde{q}\left(\zeta^{+}\right) z \leq 0\right\}
$$

Because $\bar{y}^{\ell}(J, \mathfrak{Z} ; \zeta) \leqq e$, we can replace $y$ by $\bar{y}^{\ell}(J, \mathfrak{Z} ; \zeta)$ in (76) to obtain

$$
\begin{aligned}
& w^{\ell}\left(\bar{y}^{\ell}(J, J, \mathfrak{Z} ; \zeta), \zeta^{+} ; \theta, e, J, \mathfrak{Z}\right) \\
& \quad=\max _{z}\left\{u(z+e ; \theta) \mid z \geqq-\bar{y}^{\ell}(J, \mathfrak{Z} ; \zeta) \text { and } \tilde{q}\left(\zeta^{+}\right) z \leq 0\right\}
\end{aligned}
$$

But the requirement that $y \leqq \bar{y}^{\ell}(J, \mathfrak{Z} ; \zeta)$ implies that the constraint $z \geqq-y$ in (76) is at least as restrictive as the corresponding constraint $z \geqq-\bar{y}^{\ell}(J, \mathfrak{Z} ; \zeta)$ in (77). Hence

$$
w^{\ell}\left(y, \zeta^{+} ; \theta, e, J, \mathfrak{Z}\right) \leq w^{\ell}\left(\bar{y}^{\ell}(J, \mathfrak{Z} ; \zeta), \zeta^{+} ; \theta, e, J, \mathfrak{Z}\right)
$$

Recall that the inequality (78) holds for all possible probability measures $\zeta^{+} \in$ $\mathscr{M}_{\eta}\left(L \times 2^{K} \times \mathscr{Z}^{K} \times \mathbb{R}_{+}^{G} ; \mathscr{Z}^{+}\right)$that could be estimated at Step 19. It follows that for any agent described by the triple $(\ell, J, \mathfrak{Z})$ and any measure $\zeta \in \mathscr{M}_{\gamma}^{*}$ over such triples, the compliant warehouse supply strategy with $y=\bar{y}^{\ell}(J, \mathfrak{Z} ; \zeta)$ weakly dominates any alternative $y$ satisfying the requirements that $0 \leqq y \leqq e$ and $y \leqq \bar{y}^{\ell}(J, \mathfrak{Z} ; \zeta)$.

As further intuition for (78), note that supplying any vector $y \leqq \bar{y}^{\ell}(J, \mathfrak{Z} ; \zeta)$ in effect offers the free option to repurchase some of those supplies in case this is optimal ex post given the price vector $\tilde{q}\left(\zeta^{+}\right)$that is used to clear the second submarket. For this reason, among those warehouse supply vectors that satisfy $y \leqq \bar{y}^{\ell}(J, \mathfrak{Z} ; \zeta)$, a larger vector $y$ always weakly dominates any smaller one.

\subsection{Dominant demand revelation}

A standard argument was provided in Sect. 6.6 to show that the Walrasian demand revelation strategy $\hat{\mathfrak{s}}:=\left(H(e), \hat{\mathfrak{Z}}^{H(e)}\right)=\left(H(e),\left\langle\hat{\mathfrak{Z}}_{K, t}\right\rangle_{K \in 2^{G}}\right)$ specified in (48) is dominant for each trader. That argument presumed that the final allocation would be a net demand vector in the value of the announced demand correspondence at the equilibrium price vector chosen by player 0 .

In the game being considered here, the situation is somewhat more involved. Nevertheless, after eliminating all weakly dominated strategies for each trader at Step 21, then Step 17, and finally Step 11, each trader at Steps 2 and 6 participates in what is equivalent to the game $\mathfrak{G}$ of demand revelation that was considered in Sect. 6. All traders face the common strategy set $\mathfrak{S}$ specified in (41). The pay-off of each trader with label $\ell \in L$ and type $t=(\theta, e) \in T$ can be expressed as a function

$$
w^{\ell}(\mathfrak{s}, \zeta ; \theta, e)=u\left(\bar{x}^{\ell}(J, \mathfrak{Z} ; \zeta)-\bar{y}^{\ell}(J, \mathfrak{Z} ; \zeta)+e ; \theta\right)=u\left(\bar{z}^{\ell}(J, \mathfrak{Z} ; \zeta)+e ; \theta\right)
$$

of their own strategy $\mathfrak{s} \in \mathfrak{S}$, as well as of the joint distribution $\zeta \in \mathscr{M}_{\gamma}^{*}$ of observable triples $(\ell, J, \mathfrak{Z})$. The right-hand side, of course, is exactly the same as in (44) provided that one takes $J=K(\sigma), \mathfrak{Z}=\mathfrak{Z}_{K(\sigma)}^{H}$, and $\zeta=\zeta(\sigma)$. So the truthful Walrasian demand revelation strategy $\hat{\mathfrak{s}}$ given by (48) remains the unique weakly dominant strategy in the game $\mathfrak{G}$. 


\subsection{Subgame perfect Bayesian strategyproofness}

To summarize, for each trader described by the triple $(\ell, \theta, e) \in L \times T$, one now has the following results.

- The assumption of strictly monotone preferences implies that, in the continuation game that arises after the market organizer has completed Step 20 and specified the maximum possible withdrawal vector $\tilde{z}^{\ell}\left(J, \mathfrak{Z}, y, \mathfrak{Z}^{+} ; \mu\right)$ for this trader, at Step 21 the maximal demand strategy of withdrawing this full amount is strictly dominant.

- In the continuation game that arises after the market organizer has completed Step 16 and announced that markets have to reopen, a weakly dominant strategy for the trader at Step 17 is to announce the true Walrasian demand correspondence $\mathfrak{Z}^{+} \in \mathscr{Z}^{+}$, followed by the above full withdrawal strategy at Step 21 .

- In the continuation game that arises at Step 11, after the market organizer has completed Step 10 and announced the trader's mandated warehouse supply vector $\bar{y}^{\ell}(J, \mathfrak{z}, \zeta)$, the trader's unique dominant strategy is endowment constrained compliance. That is, the supply strategy is to deposit the vector $\bar{y}_{e}^{\ell}(J, \mathfrak{Z}, \zeta)$ defined by (71). Starting at Step 17, this is followed by the above continuation strategy.

- At Step 6 of the game, when the trader is called upon to announce a first-stage regular net demand correspondence $\mathfrak{Z} \in \mathscr{Z}^{\ell}$, a weakly dominant strategy is to announce the true Walrasian net demand correspondence $\hat{\mathfrak{Z}}_{K . t}$ given the trader's true type $t$, followed by the above continuation strategy starting at Step 11.

- At Step 2 of the game, when the trader is called upon to announce an endowment set $H \subseteq G$, a weakly dominant strategy is to announce the true endowment set $H(e)$, followed by the above continuation strategy starting at Step 5.

In this sense, for our twenty-two step game in extensive form, a subgame perfect Bayesian equilibrium in unique subgame perfect dominant or weakly dominant strategies consists of the strategy profile where every trader:

1. starts by revealing the true endowment set at step 2;

2. then, at step 6, reveals the true Walrasian net demand correspondence;

3 . then, at step 11, follows the minimal compliant supply strategy in choosing a warehouse deposit;

4. then, at step 17, if markets need to reopen, reveals the new true Walrasian net demand correspondence given what was supplied at the previous stage;

5. finally, at step 21, withdraws as much from the warehouse as player 0 allows.

Of course, this strategy profile does implement the Walrasian equilibrium desired by player 0 who combines the five roles of auctioneer, clerk, statistician, principal, and warehouse manager. In every second-stage submarket, our mechanism also allows an amended Walrasian equilibrium to be reached with unique dominant or weakly dominant strategies of demand revelation and warehouse withdrawal.

Finally, following the analysis in Hammond $(1987,2011)$ as well as Guesnerie (1998), we note that the demand revelation mechanisms built around Steps 6 and 17 are multilaterally strategy proof in the sense that, for each finite coalition $\mathfrak{C} \subset L \times T$, there is no combination of misreported demand correspondences and hidden trades on the side that makes every member of $\mathfrak{C}$ better off. 


\section{Concluding remarks}

The analysis has been limited to a static system of spot markets, in which many competing traders interact with an omnipotent principal who may have no information regarding traders' preferences or endowments. Demand revelation allows Walrasian equilibrium to be reached, at least in principle, provided that one makes no concessions to computational limitations. In practice, a demand revelation process could require traders to submit a trading algorithm. This could take the form of a selection from a menu of approved standard algorithms which differ from each other depending on a variable high-dimensional parameter vector that the trader can choose.

When traders cannot trust each other to fulfil supply contracts, perhaps because endowments can be hidden and consumed away from the market, one also needs a warehouse system, along with a procedure for deterring defaulters.

\subsection{Production}

It would be routine to allow each agent to choose production activities at any combination of the following three stages:

1. before supplies are deposited in the warehouse;

2. after supplies have been deposited in the warehouse, but before demands are withdrawn;

3. after demands have been withdrawn.

It should also be routine to allow the warehouse deposits to be used as inputs in a production process that generates new outputs available for withdrawal. That would allow a system of local warehouses, between which goods can be transported at some cost.

\subsection{Other possible extensions}

Much bigger challenges are posed by:

- Financial markets, of even the simplest kind, in a two-period riskless economy with a pure credit market. Any such financial market poses the problem of enforcing promises to repay loans which cannot be secured by warehouse deposits, because some second-period commodities may not even exist in the first period. Using durable goods as collateral for loans can help alleviate these moral hazard issues, but cannot obviate them entirely.

- Labour markets, where unless employers and employees trust each other to some extent, it seems difficult to ensure subgame perfect strategyproofness without some form of coercive contract enforcement.

- Public goods financed by taxation, aggregate non-convexities in production, insurance markets with adverse selection, etc.

Acknowledgements A previous version of this paper was entitled "On Designing an Efficient Spot Market System: From Myerson, Selten and Harsanyi to Walras via Warehousing and Demand Revelation". The earlier title obviously owes much to that chosen by Dubey and Geanakoplos (2003) for their contribution 
to a symposium honouring Martin Shubik which was devoted to non-cooperative strategic market games of the kind originally considered by Shapley and Shubik (1977). The paper was first presented in April 2014 to the Cowles 10th General Equilibrium conference at Yale University and then in June 2014 to the NSF/NBER Decentralization Conference in Palo Alto, followed by the Summer Workshop in Economic Theory and associated SAET conference at the Université de Paris I (Sorbonne-Panthéon) in honour of Bernard Cornet. There were later presentations to Economics Seminars at the Universities of Hull in March 2015 and York in March 2016, to the 50th anniversary conference of the Department of Economics at the University of Essex in June 2015, to the conference in honour of Sergiu Hart at the Hebrew University in July 2015, and to the Econometric Society World Congress in Montréal, August 2015. Helpful comments from the audiences at these presentations, especially John Geanakoplos, Robert Anderson, Beth Allen, Kenneth Judd, Stefano Bosi, and Michael Wickens, are all gratefully acknowledged. So is a brief discussion with Kenneth Arrow regarding the genesis of the existence proof in Arrow and Debreu (1954). Finally, my thanks to an anonymous referee. The usual disclaimer applies.

Open Access This article is distributed under the terms of the Creative Commons Attribution 4.0 International License (http://creativecommons.org/licenses/by/4.0/), which permits unrestricted use, distribution, and reproduction in any medium, provided you give appropriate credit to the original author(s) and the source, provide a link to the Creative Commons license, and indicate if changes were made.

\section{Appendix 1: Probability in Polish spaces}

\section{Polish spaces}

Definition 3 A topological space $(U, \mathscr{U})$ is:

1. separable just in case $U$ is the closure in $\mathscr{U}$ of a countable subset of $U$;

2. metrizable just in case there is a metric $d: U \times U \rightarrow \mathbb{R}_{+}$such that $\mathscr{U}$ is generated by the family $B_{\epsilon}(u):=\{v \in U \mid d(v, u)<\epsilon\}$ of open balls, as $u$ varies over $U$ and $\epsilon$ varies over positive reals.

3. completely metrizable if it is metrizable by a metric which makes the space $U$ complete in the sense that each Cauchy sequence converges. ${ }^{7}$

A space is Polish just in case it is separable and completely metrizable.

It is easy to check that the topological product $\left(U_{1} \times U_{2}, \mathscr{U}_{1} \otimes \mathscr{U}_{2}\right)$ of two Polish spaces $\left(U_{1}, \mathscr{U}_{1}\right)$ and $\left(U_{2}, \mathscr{U}_{2}\right)$ is also Polish.

\section{The Glivenko-Cantelli theorem}

Let $(U, d)$ be any separable metric space, and let $\alpha$ be any probability measure on its Borel $\sigma$-algebra. Let $\alpha^{\mathbb{N}}$ denote the infinite product measure on the collection $U^{\mathbb{N}}$ of countably many copies of $(U, d)$, equipped with its product $\sigma$-algebra.

For each infinite sequence $u^{\mathbb{N}}=\left(u^{k}\right)_{k \in \mathbb{N}} \in U^{\mathbb{N}}$ and for each $n \in \mathbb{N}$, let

$$
\alpha_{n}\left(u^{\mathbb{N}}\right):=\frac{1}{n} \sum_{k=1}^{n} \delta_{u^{k}}
$$

\footnotetext{
7 An infinite sequence $u^{\mathbb{N}}=\left(u_{n}\right)_{n \in \mathbb{N}}$ of points $u_{n} \in U$ is a Cauchy sequence just in case, for each $\epsilon>0$, there exists $n_{\epsilon} \in \mathbb{N}$ such that, whenever both $m>n_{\epsilon}$ and $n>n_{\epsilon}$, then one has $d\left(u_{m}, u_{n}\right)<\epsilon$.
} 
denote the empirical measure on $U$ which is generated by $\left\{u^{1}, u^{2}, \ldots, u^{n}\right\}$-i.e. the first $n$ elements of $u^{\mathbb{N}}$. The Glivenko-Cantelli theorem can be stated as follows:

Theorem 1 Let $(U, d)$ be any separable metric space, and $\alpha$ be any probability measure on its Borel $\sigma$-algebra. Then, for $\alpha^{\mathbb{N}}$-a.e. $u^{\mathbb{N}} \in U^{\mathbb{N}}$, the empiricalmeasure $\alpha_{n}\left(u^{\mathbb{N}}\right)$ defined by (80) converges weakly to $\alpha$.

Following Parthasarathy (1967), whose work builds on that of Varadarajan (1958), the theorem can be seen as the implication of two prior results. First, for every Borel set $B \subseteq U$, apply the (strong) law of large numbers to the random variables defined by the indicator function $U \ni u \mapsto 1_{B}(u) \in\{0,1\}$. It implies that there exists an exceptional set $N_{B} \subset U^{\mathbb{N}}$ of sequences $u^{\mathbb{N}}$ such that $\alpha^{\mathbb{N}}\left(N_{B}\right)=0$ and, unless $u^{\mathbb{N}} \in N_{B}$, one has

$$
\alpha_{n}\left(u^{\mathbb{N}}\right)(B)=\frac{1}{n} \sum_{k=1}^{n} 1_{B}\left(u^{k}\right) \rightarrow \alpha(B)
$$

Second, because the metric space $(U, d)$ is separable, there is a countable collection $v^{\mathbb{N}}=\left\{v^{k}\right\}_{k \in \mathbb{N}}$ of points $v^{k} \in U$ such that the Borel $\sigma$-algebra of $(U, d)$ is generated by the countable collection

$$
\mathscr{B}\left(v^{\mathbb{N}}\right):=\bigcup\left\{B_{r}\left(v^{k}\right) \mid(k, r) \in \mathbb{N} \times \mathbb{Q}_{+}\right\}
$$

of open balls $B_{r}\left(v^{k}\right)$ as $k$ varies over $\mathbb{N}$ and the radius $r$ varies over the set $\mathbb{Q}_{+}$of positive rational numbers. For each $(k, r) \in \mathbb{N} \times \mathbb{Q}_{+}$, let $N_{k r}$ denote the exceptional set of sequences $u^{\mathbb{N}}$ with the property that $\alpha_{n}\left(u^{\mathbb{N}}\right)\left(B_{r}\left(v^{k}\right)\right) \not \rightarrow \alpha\left(B_{r}\left(v^{k}\right)\right)$ as $n \rightarrow \infty$. It follows that $\alpha_{n}\left(u^{\mathbb{N}}\right)(B) \rightarrow \alpha(B)$ for all Borel sets $B \in \mathscr{B}\left(v^{\mathbb{N}}\right)$ simultaneously except when $u^{\mathbb{N}}$ belongs to the $\alpha^{\mathbb{N}}$-null set $\cup_{k \in \mathbb{N}} \cup_{r \in \mathbb{Q}_{+}} N_{k r}$. It can then be shown that this convergence of measures on the countable collection $\mathscr{B}\left(v^{\mathbb{N}}\right)$ implies weak convergence.

\section{Stochastic transitions}

Starting in Sect. 5.4, the paper considers many spaces such as $\mathscr{M}_{\lambda}(L ; T)$, defined as the set of all probability measures $\tau$ on the product measurable space $(L \times T, \mathscr{B}(L \times T))$ whose marginal measure $\operatorname{marg}_{L} \tau$ on $L$ equals the fixed measure $\lambda$-i.e. $\tau(K \times T)=$ $\lambda(K)$ for every Borel set $K \subseteq L$

For every Borel set $B \subseteq L \times T$ and every $\ell \in L$, let $B_{\ell}$ denote the section $\{t \in T \mid(\ell, t) \in B\}$ of $B$.

Definition 4 A stochastic transition or measure disintegration of a probability measure $\tau \in \mathscr{M}(L \times T)$, which is also a regular conditional probability measure, is defined uniquely for $\lambda$-a.e. $\ell \in L$ as a measurable mapping $L \ni \ell \mapsto \tau_{\ell} \in \mathscr{M}(T)$ that, for every Borel set $B \subseteq L \times T$, satisfies

$$
\tau(B)=\int_{L} \tau_{\ell}\left(B_{\ell}\right) \lambda(\mathrm{d} \ell)
$$


Given any Borel set $K \subseteq L$, applying (82) to the Borel set $K \times T$ gives $\tau(K \times T)=$ $\int_{L} \tau_{\ell}\left((K \times T)_{\ell}\right) \lambda(\mathrm{d} \ell)$. But the sections $(K \times T)_{\ell}$ of the set $K \times T$ evidently satisfy $(K \times T)_{\ell}=T$ in case $\ell \in K$, but $(K \times T)_{\ell}=\emptyset$ in case $\ell \notin K$. This obviously implies that $\tau(K \times T)=\int_{K} \tau_{\ell}(T) \lambda(\mathrm{d} \ell)=\lambda(K)$. Since this is true for all Borel sets $K \subseteq L$, one has $\operatorname{marg}_{L} \tau=\lambda$.

Conversely, given any fixed Borel set $F \subseteq T$, consider the function

$$
\mathscr{L} \ni B \mapsto \lambda_{F}(B):=\tau(B \times F)
$$

It is easy to see that this defines $\lambda_{F}$ as a Borel measure on $L$. Moreover,

$$
\lambda_{F}(B)=\tau(B \times F) \leq \tau(B \times T)=\lambda(B)
$$

for all Borel sets $B \subseteq L$, and so $\lambda(B)=0 \Longrightarrow \lambda_{F}(B)=0$. It follows that the measure $\lambda_{F}$ is absolutely continuous w.r.t. the measure $\lambda$. So there exists a Radon-Nikodym derivative in the form of a Borel measurable mapping $L \ni \ell \mapsto E_{F}(\ell)$ with the property that $\tau(B \times F)=\lambda_{F}(B)=\int_{B} E_{F}(\ell) \lambda(\mathrm{d} \ell)$ for all Borel $B \subseteq L$.

Consider now the $\sigma$-algebra $\mathscr{L} \otimes\{\emptyset, T\}$ formed from the product of the $\sigma$-algebra $\mathscr{L}$ on $L$ with the trivial $\sigma$-algebra $\{\varnothing, T\}$ on $T$. Note that any function $L \times T \ni$ $(\ell, t) \mapsto f(\ell, t)$ is $\mathscr{L} \otimes\{\emptyset, T\}$ measurable if and only if there is an $\mathscr{L}$ measurable function $L \ni \ell \mapsto g(\ell)$ such that $f(\ell, t)=g(\ell)$, independent of $t$, for $\lambda$-a.e. $\ell \in L$.

After taking account of important technical details, it can now be shown that for each fixed Borel set $F \subseteq T$, the mapping $L \ni \ell \mapsto E_{F}(\ell)$ corresponds to a version of the $\mathscr{L} \otimes\{\varnothing, T\}$-measurable mapping

$$
L \times T \ni(\ell, t) \mapsto \mathbb{E}_{\tau}\left[1_{L \times F}(\ell, t) \mid \mathscr{L} \otimes\{\emptyset, T\}\right](\ell, t)
$$

that determines the conditional expectation given this product $\sigma$-algebra of the indicator function $L \times T \ni(\ell, t) \mapsto 1_{L \times F}(\ell, t) \in\{0,1\}$ defined by the requirement that its value is 1 iff $(\ell, t) \in L \times F$, or equivalently, iff $t \in F$. It can now be shown that for each fixed $\ell$, the mapping $F \mapsto E_{F}(\ell)$ is a conditional probability measure $\mathbb{P}(\cdot \mid \ell)$ on the Borel subsets of $T$. Moreover, the mapping $\ell \mapsto E_{F}(\ell)$ can be made $\mathscr{L}$-measurable for each Borel set $F \subset T$ in a way which ensures that $\tau(B)=\int_{L} E_{B_{\ell}}(\ell) \lambda(\mathrm{d} \ell)$ for all Borel $B \subset L \times T$. This implies that the mapping $L \times \mathscr{T} \ni(\ell, F) \mapsto E_{F}(\ell)$ is a version of a stochastic transition $L \times \mathscr{T} \ni(\ell, F) \mapsto \tau_{\ell}(F):=E_{F}(\ell)$ that satisfies (82).

\section{Extended probability measures are non-atomic}

Suppose that $\tau \in \mathscr{M}_{\lambda}(L ; T)$ and let $L \ni \ell \mapsto E_{F}(\ell)$ be any stochastic transition satisfying (82). Let $B$ be any Borel subset of $L \times T$ for which $\tau(B)>0$. Define the set $J:=\left\{\ell \in L \mid \tau_{\ell}\left(B_{\ell}\right)>0\right\}$. Then (82) implies that, because $\tau(B)>0$, one must have $\lambda(J)>0$. Now, we have assumed that the probability measure $\lambda$ is non-atomic. So there exists a measurable subset $H$ of $J$ such that $0<\lambda(H)<\lambda(J)$. Then the partition of $J$ into the pairwise disjoint subsets $H$ and $J \backslash H$ induces a corresponding partition of $B$ into the pairwise disjoint subsets $A:=(H \times T) \cap B$ and $B \backslash A=[(J \backslash H) \times T] \cap B$, 
whose respective probabilities satisfy

$$
\tau(A)=\int_{H} \tau_{\ell}\left(B_{\ell}\right) \lambda(\mathrm{d} \ell)>0 \text { and } \tau(B \backslash A)=\int_{J \backslash H} \tau_{\ell}\left(B_{\ell}\right) \lambda(\mathrm{d} \ell)>0
$$

It follows that $0<\tau(A)<\tau(B)$. This proves that $\tau$, like $\lambda$, is non-atomic.

\section{Appendix 2: Regular demands}

\section{True Walrasian demands are regular}

Let $K=G(\gamma) \subseteq G$ denote the trading set. Consider an individual trader with endowment vector $e \in \mathbb{R}_{+}^{K}$ and so the effective trading set $J=\left\{g \in K \mid e_{g}>0\right\}$. Let

$$
P_{K}^{J}:=\left\{p \in P_{K} \mid p^{J}>0^{J}\right\}
$$

denote the subset of the price simplex in $\mathbb{R}^{K}$ where at least one good $g \in J$ has a positive price. For the effective trading set $J$, a $J$-regular net demand correspondence was defined in Sect. 6.2 as a multifunction $P_{K}^{0} \ni p \mapsto Z(p ; \mathfrak{Z}) \subset \mathbb{R}^{K}$ satisfying:

(i) [budget exhaustion] $p z=0$ for all $p \in P_{K}^{0}$ and all $z \in Z(p ; \mathfrak{Z})$.

(ii) [feasibility]: $z \geqq-\left(\bar{e}^{J}, 0^{K \backslash J}\right)$ for all $p \in P_{K}^{0}$ and for all $z \in Z(p ; \mathfrak{Z})$.

(iii) [continuity]: The correspondence $\mathfrak{Z}$ has a graph

$$
\Gamma_{\mathfrak{Z}}:=\left\{(p, z) \in P_{K}^{0} \times \mathbb{R}^{K} \mid z \in Z(p ; \mathfrak{Z})\right\}
$$

which is a relatively closed subset of $P_{K}^{0} \times \mathbb{R}^{K}$.

(iv) [boundary condition]: If the infinite sequence $\left(p^{n}, z^{n}\right)_{n \in \mathbb{N}}$ in $\Gamma_{\mathfrak{Z}}$ is such that $p^{n}$ converges to a point $\bar{p}$ on the boundary of $P_{K}^{J}$, then the sum $\sum_{g \in K} z_{g}^{n} \rightarrow+\infty$.

Lemma 1 Let $e \in \mathbb{R}^{K}$ be any endowment vector satisfying $e \leqq \bar{e}$, let $J:=\{g \in$ $\left.K \mid e_{g}>0\right\}$ denote the associated endowment set, and let $\succsim$ be the restriction to $\mathbb{R}^{K} \times\left\{0^{G \backslash K}\right\}$ of any preference ordering on $\mathbb{R}_{+}^{G}$ that is continuous and strictly monotone. Define the extended Walrasian budget correspondence

$$
P_{K}^{J} \ni p \mapsto B^{J}(p ; e):=\left\{z \in \mathbb{R}^{K} \mid z \geqq-e \text { and } p z \leq 0\right\}
$$

along with the extended Walrasian net demand correspondence

$$
P_{K}^{J} \ni p \mapsto Z^{J}(p ; e):=\left\{z \in B^{J}(p ; e) \tilde{z} \succ z \Longrightarrow p \tilde{z}>0\right\}
$$

with graph

$$
\Gamma^{J}:=\left\{(p, z) \in P_{K}^{J} \times \mathbb{R}^{K} \mid z \in Z^{J}(p ; e)\right\}
$$

Then:

1. $Z^{J}(p ; e)=\emptyset$ if $p \in$ bd $P_{K}=P_{K} \backslash P_{K}^{0}$; 
2. $\Gamma^{J}$ is a relatively closed subset of $P_{K}^{J} \times \mathbb{R}^{K}$;

3. the restriction $\mathfrak{Z}$ of $p \mapsto Z^{J}(p ; e)$ to the subdomain $P_{K}^{0}$ is a J-regular net demand correspondence.

Proof (i) If $z \in B^{J}(p ; e)$ with $p z<0$, then there exists $\tilde{z} \gg z$ satisfying $p \tilde{z}<0$. Monotonicity implies that $\tilde{z} \succ z$, and so $z \notin Z^{J}(p ; e)$. The contrapositive implies the budget exhaustion condition requiring that $p z=0$ for every $z \in Z^{J}(p ; e)$.

(ii) The feasibility condition is an obvious implication of (86).

(iii) Suppose that $\left(p_{n}, z_{n}\right)_{n \in \mathbb{N}}$ is a sequence of points in $\Gamma^{J}$ which converges to a limit $(\bar{p}, \bar{z})$ with $\bar{p} \in P_{K}^{J}$. Then the definitions (86) and (85) evidently imply that $\bar{z} \in B^{J}(\bar{p} ; e)$. Also, because of the budget exhaustion property (i) that we have already demonstrated, one has $p_{n} z_{n}=0$ for all $n \in \mathbb{N}$. Taking the limits as $n \rightarrow \infty$, it follows that $\bar{p} \bar{z}=0$.

Consider any net trade vector $\tilde{z} \geqq-e$ satisfying $\tilde{z} \succ \bar{z}$. For any scalar $\gamma \in(0,1)$, define the convex combination

$$
z^{\gamma}:=\gamma \tilde{z}+(1-\gamma)(-e)
$$

Continuity of preferences now implies that: (a) there exists a scalar $\gamma \in(0,1)$ such that $z^{\gamma} \succ \bar{z}$; (b) $z^{\gamma} \succ z_{n}$ for all large $n \in \mathbb{N}$. Because $\left(p_{n}, z_{n}\right) \in \Gamma^{J}$ for all $n \in \mathbb{N}$, combining the definition (86) with budget exhaustion implies that $p_{n} z^{\gamma}>0=p_{n} z_{n}$ for all large $n \in \mathbb{N}$. Taking the limit as $n \rightarrow \infty$ implies that $\bar{p} z^{\gamma} \geq 0=\bar{p} \bar{z}$. By hypothesis, one has $e^{J} \gg 0^{J}$ and $\bar{p} \in P_{K}^{J}$, implying that $\bar{p}^{J}>0^{J}$ and so $\bar{p}^{J} e^{J}>0$. Because in addition $\bar{p} \bar{z}=0$, it follows that $\bar{p}(\bar{z}+e)>0$ and also, because of the definition (88), that

$$
\gamma \bar{p}(\tilde{z}-\bar{z})=\bar{p}\left[z^{\gamma}-(1-\gamma)(-e)-\gamma \bar{z}\right] \geq(1-\gamma) \bar{p}(\bar{z}+e)>0
$$

Hence $\bar{p} \tilde{z}>0$.

Finally, since this argument holds for every $\tilde{z} \succsim \bar{z}$, definition (86) implies that $\bar{z} \in Z^{J}(\bar{p} ; e)$. This proves that $\Gamma^{J}$ is a relatively closed subset of $P_{K}^{J} \times \mathbb{R}^{K}$, and also that $\Gamma_{\mathfrak{Z}}$ defined by (84) is a relatively closed subset of $P_{K}^{0} \times \mathbb{R}^{K}$.

(iv) Suppose that $\left(p_{n}, z_{n}\right)_{n \in \mathbb{N}}$ is any sequence of points in $\Gamma^{J}$ with the property that $\left(p_{n}\right)_{n \in \mathbb{N}}$ converges to a limit $\bar{p}$ on the boundary of $P_{K}^{J}$. Then $\bar{p}_{g}=0$ for at least one good $g \in K$. Hence, strict monotonicity of preferences implies that $Z^{J}(\bar{p} ; e)=\emptyset$. Because the graph $\Gamma^{J}$ of the correspondence $P_{K}^{J} \ni p \mapsto Z^{J}(p ; e)$ is a relatively closed subset of $P_{K}^{J} \times \mathbb{R}^{K}$, it follows that the sequence $\left(z_{n}\right)_{n \in \mathbb{N}}$ cannot have any limit point. In particular, it can have no convergent subsequence, and so $\sum_{g \in K}\left|z_{n, g}\right| \rightarrow \infty$ as $n \rightarrow \infty$. Because $z_{n} \geqq-e$ for all $n \in \mathbb{N}$, it follows that $\sum_{g \in K} z_{n, g} \rightarrow+\infty$ as $n \rightarrow \infty$.

\section{The space of regular net demand correspondences}

This section of the appendix uses the abbreviation $\mathrm{AB}$ to indicate the frequent page references to the book by Aliprantis and Border (1999). 
Let $\left(\mathbb{R}^{K}, \mathscr{T}\right)$ denote the finite-dimensional Euclidean space $\mathbb{R}^{K}$ with its Euclidean metric topology $\mathscr{T}$. We give an alternative characterization of regular net demand correspondences using the standard mathematical concept of the one-point compactification of $\left(\mathbb{R}^{K}, \mathscr{T}\right)$ - see $\mathrm{AB}, \mathrm{p} .56$. This is defined as the topological space $\left(\mathbb{R}_{\infty}^{K}, \mathscr{T}_{\infty}\right)$ where:

1. the set $\mathbb{R}_{\infty}^{K}:=\mathbb{R}^{K} \cup\{\infty\}$ is defined by appending to the Euclidean space $\mathbb{R}^{K}$ one extra "point at infinity" denoted by $\infty$;

2. the topology $\mathscr{T}_{\infty}$ is made up of the topology $\mathscr{T}$ of $\mathbb{R}^{K}$, together with the complement $\mathbb{R}_{\infty}^{K} \backslash V$ of each compact subset $V \subset \mathbb{R}^{K}$.

Note that each compact subset of $\left(\mathbb{R}^{K}, \mathscr{T}\right)$ remains a compact subset of $\left(\mathbb{R}_{\infty}^{K}, \mathscr{T}_{\infty}\right)$. Note too that $\left(\mathbb{R}^{K}, \mathscr{T}\right)$ is locally compact, in the sense that every point of $\mathbb{R}^{K}$ has a compact neighbourhood. It is also separable-i.e. the closure of a countable subset, such as the set $\mathbb{Q}^{K}$ of all points with rational coordinates. It follows (AB, Corollary 3.33, p. 88) that $\left(\mathbb{R}_{\infty}^{K}, \mathscr{T}_{\infty}\right)$ is metrizable. Let $d_{\infty}$ denote any metric. Then $\left(\mathbb{R}_{\infty}^{K}, d_{\infty}\right)$ is a compact metric space.

We now give the price simplex $P \subset \mathbb{R}^{K}$ the Euclidean metric $d_{P}$ of $\mathbb{R}^{K}$, and we give the Cartesian product $P \times \mathbb{R}_{\infty}^{K}$ the sup metric defined by

$$
d((p, z),(\tilde{p}, \tilde{z})):=\max \left\{d_{P}(p, \tilde{p}), d_{\infty}(z, \tilde{z})\right\}
$$

This makes $\left(P \times \mathbb{R}_{\infty}^{K}, d\right)$ a compact separable metric space.

Following (36), let $\mathscr{Z}^{K}$ denote the set of all net demand correspondences $P_{K}^{0} \ni$ $p \mapsto Z(p) \subset \mathbb{R}^{K}$ that are $J$-regular for some $J \subseteq K$. We identify each correspondence $\mathfrak{Z} \in \mathscr{Z}^{K}$ with the closure $\bar{\Gamma}_{\mathfrak{Z}}$ in the metric space $\left(P_{K} \times \mathbb{R}_{\infty}^{K}, d\right)$ of its graph $\Gamma_{\mathfrak{Z}} \subset P_{K}^{0} \times \mathbb{R}^{K}$. Because of the boundary condition (iv), this closure is easily seen to be

$$
\bar{\Gamma}_{\mathfrak{Z}}=\Gamma_{\mathfrak{Z}} \cup\left(\text { bd } P_{K} \times\{\infty\}\right)
$$

This identification establishes an injection $\mathfrak{Z} \mapsto \Gamma_{\mathfrak{Z}}$ from the set $\mathscr{Z}^{K}$ into the space $\mathscr{K}\left(P_{K} \times \mathbb{R}_{\infty}^{K}\right)$ of compact subsets of the metric space $\left(P_{K} \times \mathbb{R}_{\infty}^{K}, d\right)$. Giving the latter space its Hausdorff metric $d^{H}$ makes $\left(\mathscr{K}\left(P \times \mathbb{R}_{\infty}^{K}, d^{H}\right)\right.$ a Polish space (AB, p. 111). Then we can define the metric $d_{Z}$ on $\mathscr{Z}^{K}$ so that the distance $d_{Z}\left(\mathfrak{Z}^{\prime}, \mathfrak{Z}^{\prime \prime}\right)$ between any pair of regular demand correspondences $\mathfrak{Z}^{\prime}, \mathfrak{Z}^{\prime \prime} \in \mathscr{Z}^{K}$ equals the Hausdorff distance $d^{H}\left(\bar{\Gamma}_{\mathfrak{Z}^{\prime}}, \bar{\Gamma}_{\mathfrak{Z}^{\prime \prime}}\right)$ between the closures of their respective graphs. This construction makes $\left(\mathscr{Z}^{K}, d_{Z}\right)$ a Polish space.

\section{Appendix 3: Existence of an equilibrium price}

Here we prove existence of a market clearing equilibrium in a continuum economy when there is a well-defined measure over the set of regular net demand correspondences. The proof involves extending the arguments of, for example, Hammond (2011, Section 11, pp. 114-121) from Walrasian demand correspondences to general upper hemi-continuous demand correspondences that satisfy the Walrasian budget constraint, without necessarily corresponding to any preference-endowment pair. That 
proof in turn uses key ideas from Yamazaki $(1978,1981)$ and Khan and Yamazaki (1981).

\section{A sequence of restricted price domains}

Let $1^{K}:=(1,1, \ldots, 1)$ denote the vector in $\mathbb{R}^{K}$ whose components all equal 1 . For each $k \in \mathbb{N}$, define the non-empty price domain

$$
P_{K}^{k}:=\left\{p \in P_{K} \mid p_{g} \geq 1 /(\# K+k)(\text { all } g \in K)\right\}
$$

This is a closed subset of the relative interior $P_{K}^{0}$ of the price simplex $P$. Initially $k$ will be treated as fixed. Later, $k$ will be allowed to go to infinity in order to prove that a Walrasian equilibrium exists.

As in (25) of Sect. 5.3, assume there is a uniform upper bound $\bar{e} \in \mathbb{R}_{++}^{K}$ on traders' endowment vectors $e \in \mathbb{R}_{+}^{K}$. For each $k \in \mathbb{N}$ and $p \in P_{K}^{0}$, define the maximal budget set

$$
\bar{B}(p):=\left\{z \in \mathbb{R}^{K} \mid z \geqq-\bar{e} \text { and } p z \leq 0\right\}
$$

Then $\bar{B}(p)$ has the property that the budget set of any trader with endowment $e \in E$ satisfies

$$
B(p ; e):=\left\{z \in \mathbb{R}^{K} \mid z \geqq-e \text { and } p z \leq 0\right\} \subset \bar{B}(p)
$$

Note that for any $z \in \bar{B}(p)$ and any good $g \in K$, one has

$$
-p_{g} \bar{e}_{g} \leq p_{g} z_{g} \leq-\sum_{h \in G \backslash\{g\}} p_{h} z_{h} \leq \sum_{h \in G \backslash\{g\}} p_{h} \bar{e}_{h}
$$

Define the scalar $\omega:=\max _{g \in K}\left\{\bar{e}_{g}\right\}$. Because each $p \in P_{K}^{0}$ is normalized to satisfy $\sum_{g \in K} p_{g}=1$, Eq. (92) implies that any net trade vector $z \in \bar{B}(p)$ must satisfy

$$
p_{g} z_{g} \leq \sum_{h \in K \backslash\{g\}} p_{h} \bar{e}_{h} \leq \sum_{h \in K} p_{h} \bar{e}_{h} \leq \sum_{h \in K} p_{h} \omega=\omega
$$

and so $z_{g} \leq \omega / p_{g}$ for all $g \in K$. Whenever $p \in P_{K}^{k}$, and so $p_{g} \geq 1 /(\# K+k)$ by definition (89), it follows that $z_{g} \leq \omega(\# K+k)$ for all $g \in K$. Hence, any $z \in$ $\cup_{p \in P_{K}^{k}} B(p ; e)$ must belong to the bounded convex set

$$
\bar{B}^{k}:=\left\{z \in \mathbb{R}^{K} \mid z \geqq-\bar{e} \text { and } z \leqq \omega(\# K+k) 1^{K}\right\}
$$

So must any $z \in \cup_{p \in P_{K}^{k}} Z(p ; \mathfrak{Z})$, therefore, for any demand correspondence $\mathfrak{Z}$ which is regular because, by the definition given in (36), it belongs to the domain $\mathscr{Z}^{K}=$ $\cup_{J \subseteq K} \mathscr{Z}_{J}^{K}$.

To conclude, regularity of the demand correspondence $\mathfrak{Z}$ implies that $p \mapsto Z(p ; \mathfrak{Z})$ restricted to the domain $P_{K}^{k} \subset P_{K}^{0}$ defined by (89) has a graph $\Gamma_{\mathfrak{Z}}$ which is a closed subset of the compact set $P_{K}^{k} \times \bar{B}^{k}$, where $\bar{B}^{k}$ is defined by (93). 


\section{A convergent sequence of restricted fixed points}

Given the fixed probability measure $\zeta \in \mathscr{M}_{\gamma}^{*}$ describing the distribution of regular demand correspondences, define for each $k \in \mathbb{N}$ and associated restricted price domain $P_{K}^{k}$ the restricted mean excess demand correspondence

$$
P_{K}^{k} \ni p \mapsto F^{k}(p):=\int_{L \times 2^{K} \times \mathscr{Z} K} Z(p ; \mathfrak{Z}) \zeta(\mathrm{d} \ell \times \mathrm{d} J \times \mathrm{d} \mathfrak{Z}) \subset \mathbb{R}^{K}
$$

The integral on the right-hand side of (94) exists and is non-empty because for any price vector $p \in P_{K}^{k}$ and any regular demand correspondence $\mathfrak{Z} \in \mathscr{Z}$, the demand set $Z(p ; \mathfrak{Z})$ is a non-empty closed subset of the fixed compact set $\bar{B}^{k}$. Indeed, by Hildenbrand (1974, p. 73, Prop. 7), the integral is a compact subset of $\mathbb{R}^{K}$, and actually $F^{k}(p) \subseteq \bar{B}^{k}$ for all $p \in P_{K}^{k}$. It follows from Hildenbrand (1974, p. 73, Prop. 8) that the graph of $p \mapsto F^{k}(p)$ restricted to $P_{K}^{k}$ is also a closed subset of $P_{K}^{k} \times \bar{B}^{k}$.

Because $\operatorname{marg}_{L} \zeta=\lambda$ and the measure $\lambda$ is non-atomic, so is each distribution $\zeta \in$ $\mathscr{M}_{\gamma}^{*}$. It follows that each restricted mean excess demand correspondence $p \mapsto F^{k}(p)$ is convex-valued.

Next, for each $k=1,2, \ldots$, consider the two correspondences

$$
\bar{B}^{k} \ni z \mapsto \phi^{k}(z):=\arg \max _{p}\left\{p z \mid p \in P_{K}^{k}\right\} \subseteq P_{K}^{k}
$$

and

$$
P_{K}^{k} \times \bar{B}^{k} \ni(p, z) \mapsto \psi^{k}(p, z):=\phi^{k}(z) \times F^{k}(p) \subset P_{K}^{k} \times \bar{B}^{k}
$$

The correspondence defined by (95) has non-empty convex values throughout a domain $P_{K}^{k} \times \bar{B}^{k}$ which is the Cartesian product of two convex sets, so convex. The graph of $\psi^{k}$ is the set

$$
\Gamma_{\psi^{k}}=\left\{\left(p, z, p^{\prime}, z^{\prime}\right) \in P_{K}^{k} \times \bar{B}^{k} \times P_{K}^{k} \times \bar{B}^{k} \mid z^{\prime} \in F^{k}(p) \text { and } p^{\prime} \in \phi^{k}(z)\right\}
$$

This set is easily seen to be the Cartesian product

$$
\Gamma_{\phi^{k}} \times \Gamma_{F^{k}}=\left\{\left(z, p^{\prime}\right) \in \bar{B}^{k} \times P_{K}^{k} \mid p^{\prime} \in \phi^{k}(z)\right\} \times\left\{\left(p, z^{\prime}\right) \in P_{K}^{k} \times \bar{B}^{k} \mid z^{\prime} \in F^{k}(p)\right\}
$$

of the graph of $\phi^{k}$ with the graph of $F^{k}$, and so compact as the Cartesian product of two compact sets. Hence, the conditions for Kakutani's theorem to hold all apply. For each $k=1,2, \ldots$, therefore, the correspondence defined by (95) has a fixed point, which we denote by $\left(p^{k}, m^{k}\right) \in P_{K}^{k} \times \bar{B}^{k}$. This fixed point satisfies $\left(p^{k}, m^{k}\right) \in \psi^{k}\left(p^{k}, z^{k}\right)$ and so $p^{k} \in \phi^{k}\left(z^{k}\right), m^{k} \in F^{k}\left(p^{k}\right)$.

Note how, for all $p \in P_{K}^{k}$, the definition of $\phi^{k}$, the budget exhaustion property of regular demand correspondences implies that

$$
p m^{k} \leq p^{k} m^{k}=0
$$


Then, because the vector $(\# K)^{-1}(1,1, \ldots, 1) \in P_{K}^{k}$ for all $k \in \mathbb{N}$, this result and regularity of all the demand correspondences $\mathfrak{Z} \in \mathscr{Z}$ together imply that

$$
m^{k} \in M:=\left\{m \in \mathbb{R}^{K} \mid m \geqq-\bar{e} \text { and } \frac{1}{\# K} \sum_{g \in K} m_{k g} \leq 0\right\}
$$

Now, because each fixed point of the sequence $\left(p^{k}, m^{k}\right)_{k=1}^{\infty}$ lies in the compact subset $P \times M$ of $\mathbb{R}^{K} \times \mathbb{R}^{K}$, there must be a convergent subsequence. Retaining only the terms of this subsequence, we can assume that the sequence $\left(p^{k}, m^{k}\right)_{k \in \mathbb{N}}$ converges to some limit pair $(\hat{p}, \hat{m}) \in P \times M$.

Next, note that any $p \in P_{K}^{0}$ satisfies $p \in P_{K}^{k}$ for all large $k$, so by (96) the convergent sequence $\left(p^{k}, m^{k}\right)_{k \in \mathbb{N}}$ of fixed points in $P \times M$ must satisfy $p m^{k} \leq p^{k} m^{k}=0$ for all $k \in \mathbb{N}$. Taking limits as $k \rightarrow \infty$ yields $p \hat{m} \leq 0$ for all $p \in P_{K}^{0}$, and so

$$
\hat{m} \leqq 0
$$

\section{Positive prices}

Suppose $\left(\tilde{p}^{k}\right)_{k \in \mathbb{N}}$ is an arbitrary infinite sequence of price vectors satisfying $\tilde{p}^{k} \in P_{K}^{k}$ for each $k \in \mathbb{N}$ which converges to a limit $\tilde{p}$ on the boundary of $P$. Let $\left(\tilde{m}^{k}\right)_{k \in \mathbb{N}}$ be an associated sequence of mean net demand vectors satisfying $\tilde{m}^{k} \in F^{k}\left(\tilde{p}^{k}\right)$ for each $k \in \mathbb{N}$. Then for each $k \in \mathbb{N}$, the definition (94) implies that there is an integrable selection

$$
L \times T \ni(\ell, t) \mapsto \tilde{z}_{t k}^{\ell} \in Z\left(\tilde{p}^{k} ; \mathfrak{Z}_{t k}^{\ell, t}\right)
$$

from the values of the traders' demand correspondences at the price vector $\tilde{p}^{k}$ such that

$$
\tilde{m}^{k}=\int_{L \times 2^{K} \times \mathscr{Z} K} \tilde{z}_{t k}^{\ell} \zeta(\mathrm{d} \ell \times \mathrm{d} J \times \mathrm{d} \mathfrak{Z})
$$

But we have constructed the commodity space $K$ in order to make the mean endowment vector $\mathbb{E}_{\tau}[e]=\int_{L \times T} e \tau(\mathrm{d} \ell \times \mathrm{d} t)$ strictly positive. So obviously $\tilde{p}\left(\mathbb{E}_{\tau} e\right)=\mathbb{E}_{\tau}[\tilde{p} e]>$ 0 . It follows that there is a subset $V \subseteq L \times T$ such that $\tau(V)>0$ and $\tilde{p} e>0$ for all $(\ell, \theta, e) \in V$. Now, the net demand correspondence $\mathfrak{Z}^{\ell, t}$ of each trader with label $\ell \in L$ and type $t=(\theta, e) \in T$ is $J(e)$-regular, where $J(e)=\left\{g \in K \mid e_{g}>0\right\}$. So the boundary condition for a $J(e)$-regular net demand correspondence implies that for all $(\ell, \theta, e) \in V$ one has

$$
\sum_{g \in K} \tilde{z}_{t k g}^{\ell} \rightarrow+\infty \quad \text { as } k \rightarrow \infty
$$


But regularity also requires that $\tilde{z}_{t k}^{\ell} \geqq-\bar{e}$ for all $(\ell, t) \in L \times T$, so (98) implies that

$$
\sum_{g \in K} \tilde{m}_{k g} \geq[1-\zeta(V)] \sum_{g \in K}\left(-\bar{e}_{g}\right)+\int_{V}\left(\sum_{g \in K} \tilde{z}_{t k g}^{\ell}\right) \zeta(\mathrm{d} \ell \times \mathrm{d} J \times \mathrm{d} \mathfrak{Z})
$$

But then (99) evidently implies that

$$
\sum_{g \in K} \tilde{m}_{k g} \rightarrow+\infty \text { as } k \rightarrow \infty
$$

Now, the argument of the previous paragraphs leads to the conclusion (100) for any sequence $\left(\tilde{p}^{k}\right)_{k \in \mathbb{N}}$ of price vectors satisfying $\tilde{p}^{k} \in P_{K}^{k}$ for each $k \in \mathbb{N}$ which converges to a limit $\tilde{p}$ on the boundary of $P$. So (97) implies that, given the convergent sequence $\left(p^{k}, m^{k}\right)_{k \in \mathbb{N}}$ of fixed points in $P \times M$, the associated sequence $\left(p^{k}\right)_{k \in \mathbb{N}}$ of price vectors cannot converge to the boundary of $P$. Hence the limit price vector $\hat{p}$ must satisfy $\hat{p} \in P_{K}^{0}$.

\section{Market clearing}

We have just proved that, after choosing a subsequence if necessary, the sequence $\left(p^{k}, m^{k}\right)_{k \in \mathbb{N}}$ of fixed points converges to some pair $(\hat{p}, \hat{m}) \in P \times \mathbb{R}^{K}$ with

$$
\hat{p} \in P_{K}^{0} \quad \text { and } \quad \hat{m} \leqq 0
$$

But (96) implies that $p^{k} m^{k}=0$ for each $k \in \mathbb{N}$, so $\hat{p} \hat{m}=0$ in the limit as $k \rightarrow \infty$. Because (101) implies that $\hat{m}$ cannot have any positive components, so $\hat{m}=0$.

Finally, therefore, we can conclude that $0 \in \int_{L \times 2^{K} \times \mathscr{Z} K} Z(\hat{p} ; \mathfrak{Z}) \zeta(\mathrm{d} \ell \times \mathrm{d} J \times \mathrm{d} \mathfrak{Z})$, as required for $\hat{p}$ to be a Walrasian equilibrium price vector.

\section{Existence of Walrasian equilibrium}

Section 6 considers a spot market system where traders' endowments are observable. We have just concluded the proof that, for each $\zeta \in \mathscr{M}_{\gamma}^{*}$, there is an equilibrium price vector $p(\zeta) \in P_{K}^{0}$ along with an integrable equilibrium allocation function $L \times 2^{K} \times \mathscr{Z}^{K} \ni(\ell, J, \mathfrak{Z}) \mapsto z^{\ell}(J, \mathfrak{Z} ; \zeta)$ satisfying the requirements of Sect. 6.3. This confirms that player 0 can carry out Step 9 in the extensive form game set out in Sect. 8.1.

Finally, Sect. 7.6 considers a spot market demand revelation mechanism after each trader described by an observable pair $(\ell, J, \mathfrak{Z}) \in L \times \mathscr{Z}$ has supplied to the warehouse a vector $y \in \mathbb{R}^{K}$ satisfying $y \leqq e \wedge \bar{y}^{\ell}(J, \mathfrak{Z} ; \zeta)$, where $\bar{y}^{\ell}(J, \mathfrak{Z} ; \zeta)$ is the mandated supply vector. In effect, each trader's endowment vector has become $y$ instead of $e$.

Introducing the second-stage net trade vector $z^{+}:=x^{+}-y^{+}$with $y^{+}$fixed converts the second-stage budget constraint $q x^{+} \leq q y^{+}$set out in (58) of Sect. 7.5 to the simple constraint $q z^{+} \leq 0$ of a pure exchange economy with a trivial wealth distribution rule. 
It also converts the feasibility constraint $x^{+} \geqq 0$ to $z^{+} \geqq-y^{+}$, as in an exchange economy where $y^{+}$is the initial endowment vector. Thus, our existence proof applies to this economy also.

\section{References}

Aliprantis, C., Border, K.: Infinite Dimensional Analysis: A Hitchhiker's Guide, 2nd edn. Springer, Berlin (1999)

Arrow, K.J.: An Extension of the basic theorems of classical welfare economics. In: Proceedings of the Second Berkeley Symposium on Mathematics, Statistics, and Probability, pp. 507-532. University of California Press, Berkeley (1951)

Arrow, K.J., Debreu, G.: Existence of an equilibrium for a competitive economy. Econometrica 22(3), 265-290 (1954)

Debreu, G.: A social equilibrium existence theorem. Proc. Natl. Acad. Sci. USA 38(10), 886-893 (1952)

Dubey, P., Geanakoplos, J.: From Nash to Walras via Shapley-Shubik. J. Math. Econ. 39, 391-400 (2003)

Dubey, P., Kaneko, M.: Information patterns and Nash equilibria in extensive games: 1. Math. Soc. Sci. 8, 111-139 (1984)

Glicksberg, I.L.: A further generalization of the Kakutani fixed point theorem, with application to Nash equilibrium points. Proc. Am. Math. Soc. 3, 170-174 (1952)

Guesnerie, R.: A Contribution to the Pure Theory of Taxation. Cambridge University Press, Cambridge (1998)

Hahn, F.H.: Stability. In: Arrow, K.J., Intriligator, M. (eds.) Handbook of Mathematical Economics, Chapter 16, vol. 2, pp. 745-793. North-Holland, Amsterdam (1993)

Hammond, P.J.: Straightforward individual incentive compatibility in large economies. Rev. Econ. Stud. 46, 263-282 (1979)

Hammond, P.J.: Markets as constraints: multilateral incentive compatibility in continuum economies. Rev. Econ. Stud. 54, 399-412 (1987)

Hammond, P.J.: On the impossibility of perfect capital markets. In: Dasgupta, P., Gale, D., Hart, O., Maskin, E. (eds.) Economic Analysis of Markets and Games: Essays in Honor of Frank Hahn, pp. 527-560. M.I.T. Press, Cambridge (1992)

Hammond, P.J.: Competitive market mechanisms as social choice procedures. In: Arrow, K.J., Sen, A.K., Suzumura, K. (eds.) Handbook of Social Choice and Welfare, Chapter 15, vol. 2, pp. 47-151. NorthHolland, Amsterdam (2011)

Hammond, P.J., Sun, Y.N.: Monte Carlo simulation of macroeconomic risk with a continuum of agents: the general case. Econ. Theory 36, 303-325 (2008)

Harsanyi, J.C.: Games of incomplete information played by Bayesian players, I-III. Manag. Sci. 14, 159182, 320-334, 486-502 (1967-1968)

Hart, S., Hildenbrand, W., Kohlberg, E.: On equilibrium allocations as distributions on the commodity space. J. Math. Econ. 1, 159-166 (1974)

Hurwicz, L., Maskin, E., Postlewaite, A.: Feasible Nash implementation of social choice rules when the designer does not know endowments or production sets. In: Ledyard, J.O. (ed.) The Economics of Informational Decentralization: Complexity, Efficiency, and Stability: Essays in Honor of Stanley Reiter, Chapter 14. Kluwer Academic Press, London (1995)

Keisler, H.J.: Approximate Tâtonnement Processes. Econ. Theory 5, 127-173 (1995)

Keisler, H.J.: Getting to a competitive equilibrium. Econometrica 64, 29-49 (1996)

Khan, M.A., Yamazaki, A.: On the cores of economies with indivisible commodities and a continuum of traders. J. Econ. Theory 24, 218-225 (1981)

Koopmans, T.C.: Three Essays on the State of Economic Science. McGraw-Hill, New York (1957)

Myerson, R.B.: Optimal coordination mechanisms in generalized principal-agent problems. J. Math. Econ. 10, 67-81 (1982)

Myerson, R.B., Satterthwaite, M.A.: Efficient mechanisms for bilateral trading. J. Econ. Theory 29, 265-281 (1983)

Nash, J.F.: Equilibrium points in $n$-person games. Proc. Natl. Acad. Sci. USA 36, 48-49 (1950)

Parthasarathy, K.: Probability Measures on Metric Spaces. Academic Press, New York (1967) 
Polak, B.: Epistemic conditions for Nash equilibrium, and common knowledge of rationality. Econometrica 67, 673-676 (1999)

Postlewaite, A.: Manipulation via endowments. Rev. Econ. Stud. 46, 255-262 (1979)

Serizawa, H.: Inefficiency of strategy-proof rules for pure exchange economies. J. Econ. Theory 106, 219$241(2002)$

Serizawa, H., Weymark, J.: Efficient strategy-proof exchange and minimum consumption guarantees. J. Econ. Theory 109, 246-263 (2003)

Shapley, L., Shubik, M.: Trade using one commodity as a means of payment. J. Polit. Econ. 85, 937-968 (1977)

Varadarajan, V.S.: On the convergence of sample probability distributions. Sankhyā 19, 23-26 (1958)

Vickrey, W.: Counterspeculation, auctions, and competitive sealed tenders. J. Finance 16, 8-37 (1961)

Wilson, R.: Incentive efficiency of double auctions. Econometrica 53, 1101-1116 (1985)

Yamazaki, A.: An equilibrium existence theorem without convexity assumptions. Econometrica 46, 541555 (1978)

Yamazaki, A.: Diversified consumption characteristics and conditionally dispersed endowment distribution: regularizing effect and existence of equilibria. Econometrica 49, 639-654 (1981) 